\title{
ALGORITHMIC CONSTRUCTION OF HURWITZ MAPS
}

\author{
LAURENT BARTHOLDI, XAVIER BUFF, HANS-CHRISTIAN GRAF VON BOTHMER, \\ AND JAKOB KRÖKER
}

\begin{abstract}
We describe an algorithm that, given a $k$-tuple of permutations representing the monodromy of a rational map, constructs an arbitrarily precise floating-point complex approximation of that map.

We then explain how it has been used to study a problem in dynamical systems raised by Cui.
\end{abstract}

\section{INTRODUCTION}

Let $\mathbb{S}$ be a topological oriented 2 -sphere. The branched coverings $\mathbb{S} \rightarrow \mathbb{S}$ considered in this article are all orientation preserving. Let $Q:=\left\{Q_{i}\right\}_{i \in I}$ be a finite subset of $\mathbb{S}$ with $I=\mathbb{Z} / k \mathbb{Z}$. In [1], Hurwitz describes an elegant classification of branched coverings $\mathbb{S} \rightarrow \mathbb{S}$ with critical values contained in $Q$ in terms of admissible $k$-tuples of permutations $\left(\sigma_{i} \in \mathfrak{S}_{d}\right)_{i \in I}$. A $k$-tuple is admissible if:

- the permutations $\left(\sigma_{i}\right)_{i \in I}$ generate a transitive subgroup of $\mathfrak{S}_{d}$,

- $\sigma_{1} \cdot \sigma_{2} \cdots \sigma_{k}=$ id and

- the cycle lengths satisfy the condition

$$
\sum_{i \in I} \sum_{\begin{array}{c}
c \text { cycle } \\
\text { of } \sigma_{i}
\end{array}}(\text { length }(c)-1)=2 d-2 .
$$

See $\$ 1.1$ for more details regarding the classification.

It is easy, using a computer algebra system such as GAP [8, to enumerate all admissible $k$-tuples of permutations; it is an altogether different problem to construct an analytic model of a covering associated to a given admissible $k$-tuple of permutations. The purpose of this note is to describe such an algorithm and its implementation.

1.1. Hurwitz's classification. Two branched coverings $f: \mathbb{S} \rightarrow \mathbb{S}$ and $g: \mathbb{S} \rightarrow \mathbb{S}$ are equivalent if there is an orientation preserving homeomorphism $h: \mathbb{S} \rightarrow \mathbb{S}$ such that $g=f \circ h$. Hurwitz's result is a classification of equivalence classes of coverings in this sense.

Choose a basepoint $* \in \mathbb{S} \backslash Q$. For each $i \in I$, choose a path $\gamma_{i}$ joining $*$ to $Q_{i}$ in $\mathbb{S} \backslash Q$, in such a way that

- the paths $\gamma_{i}$ intersect only at $*$,

- the paths $\left(\gamma_{1}, \ldots, \gamma_{k}\right)$ are ordered cyclically counterclockwise around $*$.

Date: 25 October 2013.

The authors acknowledge support from the Courant Research Centre "Higher Order Structures" at Georg-August Universität zu Göttingen and the CIMI at Toulouse. 
The fundamental group $G=\pi_{1}(\mathbb{S} \backslash Q, *)$ is generated by paths $\hat{\gamma}_{i}$ that follow $\gamma_{i}$, wind once counterclockwise around $Q_{i}$, and return to $*$ along $\gamma_{i}$. It has the presentation

$$
G=\left\langle\hat{\gamma}_{i}, i \in I \mid \hat{\gamma}_{1} \cdot \hat{\gamma}_{2} \cdots \hat{\gamma}_{k}=\mathrm{id}\right\rangle
$$

Let $f:(\mathbb{S}, C) \rightarrow(\mathbb{S}, Q)$ be a covering branched over $Q$. Number $\left\{*_{1}, \ldots, *_{d}\right\}$ the $f$-preimages of $*$. Then, for each $i \in I$ and each $m \in\{1, \ldots, d\}$, the path $\hat{\gamma}_{i}$ lifts to a path starting at $*_{m}$ and ending at $*_{n}$ for some $n=: \sigma_{i}(m)$. This defines a permutation $\sigma_{i}$ for each $i \in I$. Note that the $k$-tuple $\left(\sigma_{i}\right)_{i \in I}$ is admissible:

- since $\mathbb{S} \backslash C$ is connected, the group $\left\langle\sigma_{i}\right\rangle$ is transitive on $\{1, \ldots, d\}$;

- since $\hat{\gamma}_{1} \cdot \hat{\gamma}_{2} \cdots \hat{\gamma}_{k}=\mathrm{id}$, we have that $\sigma_{1} \cdot \sigma_{2} \cdots \sigma_{k}=\mathrm{id}$

- computing the Euler characteristic of $\mathbb{S} \backslash C$ via the Riemann-Hurwitz formula yields (1).

Conversely, let $\left(\sigma_{i}\right)_{i \in I}$ be an admissible $k$-tuple of permutations. Define a branched covering as follows: start with $d$ disjoint copies of $\mathbb{S}$, cut open along the paths $\gamma_{i}$. If $\sigma_{i}(m)=n$, glue the right boundary of $\gamma_{i}$ on $m$-th sphere to the left boundary of $\gamma_{i}$ on the $n$-th sphere. This defines a covering with critical values contained in $Q$. It is connected because $\left\langle\sigma_{i}\right\rangle$ is transitive on $\{1, \ldots, d\}$. The Euler characteristic of the cover is 2, because of (1) and the Riemann-Hurwitz formula; so it is a sphere.

The $k$-tuple $\left(\sigma_{i}\right)_{i \in I}$ must be considered up to diagonal conjugation by $\mathfrak{S}_{d}$, which amounts to numbering the spheres differently. The constructions above then define a bijection between equivalence classes of branched coverings and equivalence classes of appropriate $k$-tuples of permutations.

A coarser equivalence relation on coverings has also been considered, but is not the main focus of this article: two coverings $f, g: \mathbb{S} \rightarrow \mathbb{S}$ are Hurwitz equivalent if there exist homeomorphisms $h_{0}, h_{1}: \mathbb{S} \rightarrow \mathbb{S}$ with $f \circ h_{1}=h_{0} \circ g$. Hurwitz classes of coverings may also be classified by $k$-tuples of permutations; namely, by the orbits on appropriate $k$-tuples of the symmetric group $\mathfrak{S}_{d}$ (acting as above) and the pure braid group on $k$ strings. The latter group's generators act by conjugating, for any two consecutive points $Q_{i}, Q_{i+1}$ in $Q$, the permutations $\sigma_{i}$ and $\sigma_{i+1}$ by $\sigma_{i} \sigma_{i+1}$. This amounts to changing the "spider" $\bigcup_{i \in I} \gamma_{i}$ by twisting the legs $\gamma_{i}$ and $\gamma_{i+1}$ around each other.

1.2. Analytic models. Assume now $Q \subset \mathbb{P}^{1}(\mathbb{C})$ and that $f: \mathbb{S} \backslash C \rightarrow \mathbb{P}^{1}(\mathbb{C}) \backslash Q$ is a covering map. Then, $f$ defines holomorphic charts on $\mathbb{S} \backslash C$ and it is not difficult to see that the points in $C$ are removable singularities: we denote by $\mathbb{S}_{f}$ the corresponding Riemann surface. By the Uniformization Theorem, there is a conformal homeomorphism $\phi_{f}: \mathbb{S}_{f} \rightarrow \mathbb{P}^{1}(\mathbb{C})$. The map $F:=f \circ \phi_{f}^{-1}: \mathbb{P}^{1}(\mathbb{C}) \rightarrow$ $\mathbb{P}^{1}(\mathbb{C})$ is a holomorphic branched covering, i.e., a rational map. Assume $g=f \circ$ $h: \mathbb{S} \rightarrow \mathbb{P}^{1}(\mathbb{C})$ for some homeomorphism $h: \mathbb{S} \rightarrow \mathbb{S}$. Let $\phi_{g}: \mathbb{S}_{g} \rightarrow \mathbb{P}^{1}(\mathbb{C})$ be a conformal homeomorphism and set $G:=g \circ \phi_{g}^{-1}: \mathbb{P}^{1}(\mathbb{C}) \rightarrow \mathbb{P}^{1}(\mathbb{C})$ be the corresponding rational map. Then, $H=\phi_{f} \circ \phi_{g}^{-1}: \mathbb{P}^{1}(\mathbb{C}) \rightarrow \mathbb{P}^{1}(\mathbb{C})$ is a Möbius transformation and $F=G \circ H$.

Therefore, up to precomposition by a Möbius transformation, the rational map $F$ only depends on the equivalence class of covering $f:(\mathbb{S}, C) \rightarrow\left(\mathbb{P}^{1}(\mathbb{C}), Q\right)$. We say that $f$ is an analytic model.

1.3. Dynamics. Our algorithm is an important step in the more difficult problem of determining an analytic model with given dynamics. We start by recalling some 
definitions. The post-critical set of a branched self-covering $f: \mathbb{S} \rightarrow \mathbb{S}$ with critical value set $Q_{f}$ is

$$
P_{f}:=\bigcup_{n \geq 0} f^{\circ n}\left(Q_{f}\right) .
$$

We are interested in the case where $P_{f}$ is finite and we consider $f$ up to isotopy rel $P_{f}$; namely, we that that $f$ and $g$ are combinatorially equivalent, and write $f \sim g$, if there exists a path of branched self-coverings from $f$ to $g$ whose post-critical set moves smoothly.

The dynamical problem alluded to above asks to determine, given a branched covering $f: \mathbb{S} \rightarrow \mathbb{S}$ with finite post-critical set, whether there exists a rational map that is combinatorially equivalent to $f$, and in that case to exhibit such a rational map.

A fundamental theorem of Thurston (see [7] and Theorem 8.1 below) proves (except in few well-understood, low-complexity cases) that such a branched covering $f$ is combinatorially equivalent to at most one rational map, up to conjugation by a Möbius transformation; furthermore, if $\# Q=3$, then it has precisely one holomorphic realization.

In case $f$ is a topological polynomial (it has a fixed point of maximal ramification), the dynamics of $f$ may be described by combinatorial data called "external rays", see [19. An implementation, when $f$ has only two critical values, is described in [10, and is called the "spider algorithm"; see also [4] treating the general degree2 case. In a forthcoming paper, the first author will describe the implementation of the general case.

If $\# Q=3$, then we may assume $Q_{1}=\infty, Q_{2}=0$ and $Q_{3}=1$ within $\mathbb{P}^{1}(\mathbb{C})$. Furthermore, precomposing $f$ by an appropriate Möbius transformation, we may also assume that $Q \subset f^{-1}(Q)$. In the polynomial case, Pilgrim linked in 18 the "dessin d'enfant" (the full preimage of the segment $[0,1]$ ) of $f$ with a dynamical invariant, its "Hubbard tree".

We describe in Section 8 a question by Cui in the theory of holomorphic dynamical systems, and give an explicit holomorphic realization of a topological map he constructed.

This will also be our running example in the text. With $Q_{1}=\infty, Q_{2}=0$ and $Q_{3}=1$, the permutations representing the map are

$$
\begin{aligned}
& \sigma_{1}=(1,7,11,2)(3,8) \underline{(4,5)}(6,10)(9,12,13), \\
& \sigma_{2}=(1,3,12,4)(5,9) \underline{(6,7)}(10,13,11)(2,8), \\
& \sigma_{3}=(1,5,13,6)(7,10) \underline{(2,3)}(8,11,12)(4,9) .
\end{aligned}
$$

Recall that the cycles of the above permutations correspond to preimages of critical values. We seek a degree-13 rational map $f$ such that the underlined cycle $(4,5)$ and its image under $f$ are located at $\infty$, and similarly for the other two cycles and images.

In this specific example, the search can be made more feasible as follows. Setting all critical points as unknowns and eliminating is out of the question. With a little faith that the symmetry between $\infty, 0,1$ translates to $f$, let $\rho(z)=1 /(1-z)$ be the rotation permuting $\infty, 0,1$, and note that $\mathbb{P}^{1}(\mathbb{C}) /\langle\rho\rangle$ is a sphere, branched at the two fixed points of $\rho$. If $f$ descends to a map $g$ on $\mathbb{P}^{1}(\mathbb{C}) /\langle\rho\rangle$, then (after change of variables) it has the form $g(z)=z(p(z) / q(z))^{3}$ for degree- 4 polynomials, such that $g(z)=1+\mathcal{O}\left((z-1)^{4}\right)$ at $z=1$, and such that 1 is the image of four other points 
with local degrees $3,2,2,2$ respectively. We are grateful to Noam Elkies and Curt McMullen for having pointed out to us the feasibility of this approach.

Nevertheless, we will show that our algorithm is strong enough to produce a solution even without exploiting the symmetry of the Hurwitz data.

1.4. Simple cases. If $\# Q=2$, then there is a unique solution represented, up to diagonal conjugation, by the pair of permutations

$$
\sigma_{1}=(1,2, \ldots, d) \quad \text { and } \quad \sigma_{2}=(d, \ldots, 2,1) .
$$

If $Q_{1}=\infty$ and $Q_{2}=0$, an analytic model is $f(z)=z^{d}$.

However, the case $\# Q=3$ seems already as complicated as the general case, and has only been addressed in the literature for small $d$. Such maps are often called "dessins d'enfant", see [9]; the corresponding combinatorial objects for the modular surface $\mathfrak{h} / \mathrm{PSL}_{2}(\mathbb{Z})$ are called "Conway diagrams", see [1, §3.4]. Methods of constructing them are addressed, inter alia, in $3,5,6$.

In this section, we consider the case $d \leq 3$ which can completely be solved. If $\# Q=2$, then as we said above we may choose $Q=\{\infty, 0\}$ and $f(z)=z^{d}$. If $d=\# Q=3$ then we may choose $Q=\{\infty, 0,1\}$. Without loss of generality, we may assume that all points of $Q$ are branched values, since otherwise we are reduced to the case $\# Q=2$. Up to permutation of the points in $Q$ and the indices, the only possible triple of permutations is

$$
\sigma_{1}=(1,2,3), \quad \sigma_{2}=(1,2) \quad \text { and } \quad \sigma_{3}=(2,3) .
$$

To find an analytic model, we seek a rational map $f$ of degree 3 such that

$$
\infty \stackrel{3: 1}{\mapsto} \infty, \quad 0 \stackrel{2: 1}{\mapsto} 0 \quad \text { and } \quad 1 \stackrel{2: 1}{\mapsto} 1 \text {. }
$$

This implies $f(z)=3 z^{2}-2 z^{3}$ as the only realization.

The next case we consider is $d=3$ and $\# Q=4$. Using Möbius transformations, we may normalise $Q$ to be $\{\infty, 0,1, w\}$. Up to conjugation in $\mathfrak{S}_{3}$ we may take the first permutations to be $\sigma_{1}=\ldots=\sigma_{i}=(1,2)$. The condition that the permutations generate a transitive group imply that one of them is not $(1,2)$. Up to conjugation, we may assume that the first permutation which is not $(1,2)$ is $\sigma_{i+1}=(2,3)$. Since $\sigma_{1} \cdot \sigma_{2} \cdot \sigma_{3} \cdot \sigma_{4}=\mathrm{id}$, this gives four possibilities, namely, writing $\sigma=\left(\sigma_{1}, \sigma_{2}, \sigma_{3}, \sigma_{4}\right)$,

$$
\begin{array}{llrl}
\sigma & =((1,2),(1,2),(2,3),(2,3)), & & \sigma=((1,2),(2,3),(1,2),(1,3)), \\
\sigma & =((1,2),(2,3),(1,3),(2,3)), & & \sigma=((1,2),(2,3),(2,3),(1,2)) .
\end{array}
$$

To find the corresponding $f$, assume without loss of generality that $f$ maps $\infty \mapsto \infty$, $0 \mapsto 0,1 \mapsto 1$ and $v \mapsto w$. This forces the map $f$ to have the form

$$
f_{a}(z)=z^{2} \frac{a(z-1)+1}{(a+2)(z-1)+1},
$$

for some parameter $a$ subject to $(a+1)(a-1)^{3}=w a(a+2)^{3}$; then $(a+1)(a-1)=$ $v a(a+2)$. Since $w \neq 0,1$, the equation defining $a$ in terms of $w$ has four distinct roots, leading to four candidate maps $f_{a}$. There is a bijection between the maps $f$ and the triples of permutations above, but no canonical one - it will depend on the specific choice of $\# Q$ generators $\hat{\gamma}_{i}$ of $\pi_{1}\left(\mathbb{P}^{1}(\mathbb{C}) \backslash Q, *\right)$. Note also that these four solutions are part of a single Hurwitz class. 
1.5. Reddite Cæsare. Various methods have already been considered for the computation of branched coverings, at least under some restrictions on the data. Note, first, that a head-on approach, solving numerically the equations after having converted them to a Gröbner basis, works only for the most simple examples, and in particular is completely unrealistic for the degree- 13 example described in $\$ 1.3$.

In case $k=3$ and $Q=\{0,1, \infty\}$, the covering is called a Belyi map; if furthermore $\sigma_{3}$ is a $d$-cycle, then the covering is called a Belyi polynomial. The explicit construction of Belyi maps has been addressed by numerous authors. Couveignes and Granboulan describe in [5] a method based on writing Puiseux series for the solution, after having made initial guesses on the positions of the roots; they obtain in this manner very high-precision approximations of the coëfficients of the map, which allow the determination of their minimal field of definition (they credit the idea to Oesterlé).

Matiyasevich conducted in [17 some experiments, and showed that Belyi polynomials can be efficiently computed by an iterative process, increasing the polynomial degree and adjusting the critical values by Newton's method. The idea is to iteratively deform the polynomial $z^{d}$ so as to obtain arbitrary critical values.

A much more efficient approach has been developed recently by Marshall and Rohde [16, and is based on the zipping algorithm 12. Zipping is much faster, and lets one construct Belyi maps of very high degree. In particular, Marshall and Rohde managed to describe all Belyi polynomials of degree $\leq 14$. They have been able to reproduce the computations in this article using their method.

\section{OVERVIEW OF THE ALGORITHM}

We are given a list $\sigma_{1}, \ldots, \sigma_{k}$ of permutations in $\mathfrak{S}_{d}$ with product $\sigma_{1} \cdots \sigma_{k}=1$, and points $Q_{1}, \ldots, Q_{k} \in \mathbb{P}^{1}(\mathbb{C})$.

Let $\alpha_{i}=\left(\alpha_{i, 1}, \ldots, \alpha_{i, \ell_{i}}\right)$ be the cycle lengths of $\sigma_{i}$; we have $\sum_{j} \alpha_{i, j}=d$ for all $i$, and $\sum_{i, j}\left(\alpha_{i, j}-1\right)=2 d-2$. In the first part of the algorithm, we enumerate all rational maps with critical values $Q_{1}, \ldots, Q_{k}$ such that the multiplicities of the preimages of $Q_{i}$ are $\alpha_{i, 1}, \ldots, \alpha_{i, \ell_{i}}$. In the second part, we select the appropriate rational map among these candidates.

The approach in the first part of the algorithm seems to originate in Malle [14]; see also [15].

For the sake of describing its workflow more clearly, the actual algorithm (described in the remainder of the text) has been slightly simplified.

Normalization: Without loss of generality, we assume $Q_{1}=\infty, Q_{2}=0$ and $Q_{3}=1$. We approximate the other $Q_{i}$ by $\tilde{Q}_{i} \in \mathbb{P}^{1}(\overline{\mathbb{Q}})$. The rational map we seek will leave $Q_{1}, Q_{2}, Q_{3}$ fixed. Using this normalization, if all $Q_{i}$ are algebraic then the coëfficients of the map will also be algebraic.

Finite field solution: We pick a prime $p$, such that the points $\tilde{Q}_{i}$ have distinct realizations $\bar{Q}_{i} \in \mathbb{P}^{1}\left(\mathbb{F}_{p}\right)$. We then list all degree- $d$ rational maps $\bar{F}$ over $\mathbb{F}_{p}$ with poles and zeroes of multiplicities $\alpha_{1}$ and $\alpha_{2}$ respectively, and by brute force check for each $\bar{F}$ whether $\bar{F}-\bar{Q}_{i}$ has zeroes of multiplicities $\alpha_{i}$ for all $i \geq 3$. Note that the rational map $\bar{F}$ is a solution to our original problem over $\mathbb{F}_{p}$. (If there are no solutions, we restart with a different prime $p$ ).

p-adic solution: Write $\bar{F}=\bar{W}_{2} / \bar{W}_{1}$ with $\bar{W}_{2}$ monic of degree $d$, and $\bar{W}_{1}$ of degree less than $d$. (In fact, we later write the denominator as $\lambda W_{1}$ 
with $W_{1}$ monic. The present discussion uses a simplified notation.) For $i \geq 3$, let $\bar{W}_{i}=\bar{W}_{2}-\bar{Q}_{i} \bar{W}_{1}$ be the numerator of $\bar{F}-\bar{Q}_{i}$. We compute high-precision $p$-adic approximations $\hat{Q}_{i}$ of the $\tilde{Q}_{i}$, and lift each $\bar{W}_{i}$ from $\mathbb{F}_{p}$ to a high-precision polynomial $\hat{W}_{i}$ over $\mathbb{Z}_{p}$, in such a manner that we have $\hat{W}_{i}=\hat{W}_{2}-\hat{Q}_{i} \hat{W}_{1}+O\left(p^{N}\right)$ for large $N$. This lifting can be done by Hensel's lemma, because by Corollary 3.3 , the Jacobian of the system $\left\{W_{i}=W_{2}-Q_{i} W_{1}\right\}$ is invertible at a solution for almost every prime $p$. (If $D \bar{F}$ happens not to be invertible, we restart with a different prime).

Algebraic solution: Using the lattice-reduction algorithm LLL [13], we find polynomials $W_{i}$ over $\overline{\mathbb{Q}}$, with coëfficients of small height (small degree and coëfficients of minimal polynomial) that are close to $\hat{W}_{i}$ obtained at the previous step. Using exact arithmetic over $\overline{\mathbb{Q}}$, we check that the solution $W_{2} / W_{1}$ is correct. (If not, we either compute a finer $p$-adic approximation, or higher-degree algebraic number approximations, or we restart altogether with a larger prime).

Complex solution: For each coëfficient $c_{i, j} \in \overline{\mathbb{Q}}$ of $W_{i}$, given by its minimal polynomial over $\mathbb{Q}$, we compute (to high, user-specified precision) all the roots $\tilde{c}_{i, j, s}$ of its minimal polynomial, as floating-point complex numbers. Not all choices of $\tilde{c}_{i, j, s}$ are compatible: there may exist some extra constraints between one coëfficient and another (such as, for example, that they are complex conjugates of each other). We determine these extra constraints as follows: we choose small, random integers $m, n$, compute the minimal polynomial of $m c_{i, j}+n c_{i^{\prime}, j^{\prime}}$, and compute (again to high precision) its roots $\tilde{d}_{i, j, i^{\prime}, j^{\prime}, t}$. We then pair together those roots $\left(\tilde{c}_{i, j, s}, \tilde{c}_{i^{\prime}, j^{\prime}, s^{\prime}}\right)$ for which $\tilde{d}_{i, j, i^{\prime}, j^{\prime}, t} \approx m \tilde{c}_{i, j, s}+n \tilde{c}_{i^{\prime}, j^{\prime}, s^{\prime}}$ for some $t$. By considering enough of these pairs we can stitch together a collection of compatible coëfficient approximations $\tilde{c}_{i, j, s}$ embracing all $i, j$.

We call $\widetilde{C}$ the collection of all coëfficients $\tilde{c}_{i, j, s}$, and note that the rational map is determined by its zeroes, its poles, and the normalization condition that $Q_{1}=1$ is fixed. Since $\{0, \infty\} \subset Q$, these zeroes and poles are determined by $\widetilde{C}$.

The second step of the algorithm checks, by path lifting, that the monodromy around $Q_{i}$ is correct. For each of the Galois conjugate solutions $(\widetilde{C}, \widetilde{f})$ obtained in the first step, we do the following:

Triangulate: We are given a floating-point approximation $\widetilde{Q}$ of $Q$. We compute a triangulation $\mathscr{Q}$ of $\mathbb{P}^{1}(\mathbb{C})$ whose vertex set contains $\widetilde{Q}$, and a triangulation $\mathscr{C}$ of $\mathbb{P}^{1}(\mathbb{C})$ whose vertex set contains $\widetilde{C}$. For efficiency reasons, we use Delaunay triangulations, see $\$ 7$. We compute the dual triangulation $\mathscr{Q}^{\perp}$; it has one vertex per face of $\mathscr{Q}$, and edges transverse to those of $\mathscr{Q}$. We fix a vertex $* \in \mathscr{Q}^{\perp}$ as our basepoint.

Lift the triangulation: Let $W$ denote the vertices of $\mathscr{Q}^{\perp}$. For each $w \in W$, we number arbitrarily $w_{1}, \ldots, w_{d}$ the $\tilde{f}$-preimages of $w$.

For each edge $\varepsilon \in \mathscr{Q}^{\perp}$, going from $w^{\prime}$ to $w^{\prime \prime}$, we compute a permutation $\varsigma_{\varepsilon} \in \mathfrak{S}_{d}$ such that the $\widetilde{f}$-lift of $\varepsilon$ starting at $w_{i}^{\prime}$ ends at $w_{\varsigma_{\varepsilon}(i)}^{\prime \prime}$. There are two strategies for this, one is by subdividing appropriately the path $\varepsilon$ and playing "connect-the-dots", the other uses more efficiently the triangulation $\mathscr{C}$. 
Read permutations: For each critical value $Q_{i} \in Q$, let $\varepsilon(1), \ldots, \varepsilon(n)$ be the sequences of edges traversed by a path in $\mathscr{Q}^{\perp}$ that starts and ends in the basepoint $*$, and surrounds once counterclockwise the point $Q_{i}$ and no other vertex of $Q$. Compute the permutation $\sigma_{i}^{\prime}=\varsigma_{\varepsilon(1)} \cdots \varsigma_{\varepsilon(n)}$.

Check: The data $(C, f)$ are a valid solution to the Hurwitz problem if and only if there exists a permutation $\tau \in \mathfrak{S}_{d}$ such that $\sigma_{i}^{\prime}=\left(\sigma_{i}\right)^{\tau}$.

2.1. Implementation. The fourth-named author has implemented the first part of the algorithm, mainly in $\mathrm{C}$, and the first-named author has implemented the second part of the algorithm, mainly in GAP 8 . By far the most time-consuming part of the procedure is the search for a solution over a finite field. Example 4.9 required approximately 15 minutes on a desktop, 30-SPECint2006 computer. The code is maintained by the fourth-named author, and is available at

\section{https://github.com/jakobkroeker/HMAC}

\section{THE SPACE OF RATIONAL MAPS}

We show, in this section, that (as soon as the prime $p$ is sufficiently large) we may lift every $\mathbb{F}_{p}$-solution to $\mathbb{Z}_{p}$. This follows from the well known fact that the Hurwitz spaces are smooth. We could not find the precise statement we need in the literature, so we give a complete proof.

Let $d \geq 2$ be an integer and denote by Rat $_{d}$ the space of rational maps of degree $d$, which may be identified with a Zariski open subset of $\mathbb{P}^{2 d+1}(\mathbb{C})$.

Let $k \geq 3$ be an integer and let $F: \mathbb{S} \rightarrow \mathbb{S}$ be a ramified covering branched over $Q=\left\{Q_{1}, \ldots, Q_{k}\right\}$. Note that, according to the Riemann-Hurwitz Formula, $C:=F^{-1}(Q)$ contains exactly $(k-2) d+2$ points. We write $C=\bigcup_{i} C_{i}$ with $C_{i}:=F^{-1}\left\{Q_{i}\right\}=\left\{C_{i, 1}, \ldots, C_{i, \ell_{i}}\right\}$, and for each $j \in\left\{1, \ldots, \ell_{i}\right\}$ we let $\alpha_{i, j}$ be the local degree of $F$ at $C_{i, j}$.

Let $\mathfrak{Q}$ be the smooth quasiprojective variety of injective maps $\mathfrak{q}: Q \rightarrow \mathbb{P}^{1}(\mathbb{C})$. For $\mathfrak{q} \in \mathfrak{Q}$, we use the notation $q_{i}:=\mathfrak{q}\left(Q_{i}\right)$. Similarly, let $\mathfrak{C}$ be the smooth quasiprojective variety of injective maps $\mathfrak{c}: C \rightarrow \mathbb{P}^{1}(\mathbb{C})$. For $\mathfrak{c} \in \mathfrak{C}$, we use the notation $c_{i, j}:=\mathfrak{c}\left(C_{i, j}\right)$. The quasiprojective variety $\mathfrak{Y}:=\mathfrak{C} \times \mathfrak{Q}$ is smooth. We shall prove that the subvariety

$$
\mathfrak{X}:=\left\{(\mathfrak{c}, \mathfrak{q}) \in \mathfrak{Y} \mid\left(\exists f \in \operatorname{Rat}_{d}\right)(\forall i, j) f\left(c_{i, j}\right)=q_{i} \text { and } \operatorname{deg}_{c_{i, j}} f=\alpha_{i, j}\right\}
$$

is also smooth, and regularly parametrised:

Proposition 3.1. The variety $\mathfrak{X}$ is smooth of dimension $k+3$, locally regularly parametrised by $\left(q_{1}, \ldots, q_{k}, c_{1,1}, c_{2,1}, c_{3,1}\right)$.

Observe that, for $(\mathfrak{c}, \mathfrak{q}) \in \mathfrak{X}$, there is a unique rational map $f \in \mathrm{Rat}_{d}$ such that $f\left(c_{i, j}\right)=q_{i}$ and $\operatorname{deg}_{c_{i, j}} f=\alpha_{i, j}$ for all $(i, j)$. Indeed, knowing a rational map above three points completely determines the rational map (it is even enough to know the full preimage of two points plus one preimage of a third point).

Note that the group of Möbius transformations acts on $\mathfrak{C}$ and $\mathfrak{Q}$ by postcomposition:

$$
(M, N) \cdot(\mathfrak{c}, \mathfrak{q}):=(M \circ \mathfrak{c}, N \circ \mathfrak{q}) .
$$

The quotient space may be identified with $\mathfrak{Y}_{0}:=\mathfrak{C}_{0} \times \mathfrak{Q}_{0}$ with

$$
\mathfrak{C}_{0}:=\left\{\mathfrak{c} \in \mathfrak{C} \mid c_{1,1}=\infty, c_{2,1}=0 \text { and } c_{3,1}=1\right\}
$$


and

$$
\mathfrak{Q}_{0}:=\left\{\mathfrak{q} \in \mathfrak{Q} \mid q_{1}=\infty, q_{2}=0 \text { and } q_{3}=1\right\} .
$$

The projection $\mathfrak{Y} \rightarrow \mathfrak{Y} /\left(\mathrm{PSL}_{2}(\mathbb{C}) \times \mathrm{PSL}_{2}(\mathbb{C})\right) \cong \mathfrak{Y}_{0}$ is a submersion.

The action preserves $\mathfrak{X}$ as indicated on the following commutative diagram:

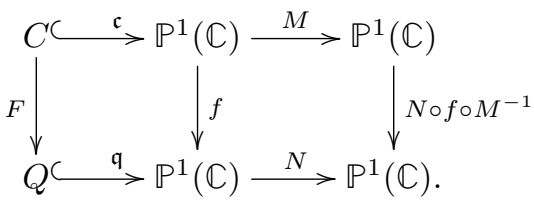

It is therefore enough to show that $\mathfrak{X}_{0}:=\mathfrak{X} \cap \mathfrak{Y}_{0}$ is a smooth subvariety of $\mathfrak{Y}_{0}$ locally regularly parametrised by $\left(q_{4}, \ldots, q_{k}\right)$.

We first write equations for $\mathfrak{X}_{0}$. To each $(\mathfrak{c}, \mathfrak{q}, \lambda) \in \mathfrak{C}_{0} \times \mathfrak{Q}_{0} \times \mathbb{C}^{*}$, we associate a collection of monic polynomials $\left(W_{i}\right)_{i \in\{1, \ldots, k\}}$ defined by

$$
W_{1}(z):=\prod_{j=2}^{\ell_{1}}\left(z-c_{1, j}\right)^{\alpha_{1, j}} \quad \text { and for } i \geq 2 \quad W_{i}(z):=\prod_{j=1}^{\ell_{i}}\left(z-c_{i, j}\right)^{\alpha_{i, j}}
$$

and a collection of rational maps $\left(f_{i}\right)_{i \in\{2, \ldots, k\}}$ defined by

$$
f_{i}:=\frac{W_{i}}{\lambda W_{1}}+q_{i}
$$

Note that these are degree- $d$ rational maps with poles of order $\alpha_{1, j}$ at $c_{1, j}$. In addition, $f_{i}$ maps $c_{i, j}$ to $q_{i}$ with local degree $\alpha_{i, j}$. It follows that $(\mathfrak{c}, \mathfrak{q}) \in \mathfrak{X}_{0}$ if and only if there is a $\lambda \in \mathbb{C}^{*}$ such that $f_{i}=f_{2}$ for all $i \in\{3, \ldots, k\}$, that is, $F_{i}=0$ with

$$
F_{i}:=W_{i}+\lambda q_{i} W_{1}-W_{2} .
$$

In that case, we use the notation

$$
f_{(\mathfrak{c}, \mathfrak{q}, \lambda)}:=f_{2}=f_{3}=\cdots=f_{k} .
$$

In other words, consider the map

$$
\mathcal{F}:=\left(F_{3}, \ldots, F_{k}\right): \mathfrak{Y}_{0} \times \mathbb{C} \rightarrow\left(\mathbb{C}[z]_{\operatorname{deg}<d}\right)^{k-2} .
$$

Then, $(\mathfrak{c}, \mathfrak{q}) \in \mathfrak{X}_{0}$ if and only if there is a $\lambda \in \mathbb{C}^{*}$ such that $\mathcal{F}(\mathfrak{c}, \mathfrak{q}, \lambda)=0$.

According to the following Lemma and the Implicit Function Theorem, the subvariety of $\mathfrak{Y}_{0} \times \mathbb{C}$ defined by the equation $\mathcal{F}=0$ is smooth of dimension $k-3$, locally regularly parametrised by $\left(q_{4}, \ldots, q_{k}\right)$. It follows that its projection to the $\mathfrak{Y}_{0}$ component, namely $\mathfrak{X}_{0}$, is also smooth of dimension $k-3$, locally regularly parametrised by $\left(q_{4}, \ldots, q_{k}\right)$.

Lemma 3.2. If $\mathcal{F}(\mathfrak{c}, \mathfrak{q}, \lambda)=0$, then the derivative $D_{(\mathfrak{c}, \mathfrak{q}, \lambda)} \mathcal{F}$ restricts to an isomorphism $T_{\mathfrak{c}} \mathfrak{C}_{0} \times\{0\} \times T_{\lambda} \mathbb{C} \rightarrow T_{0}\left(\mathbb{C}[z]_{\operatorname{deg}<d}\right)^{k-2}$.

We postpone the proof of the lemma to Section 3.2 and mention immediately a corollary that we shall use later. For $\mathfrak{q} \in \mathfrak{Q}$, let $\mathcal{F}_{\mathfrak{q}}: \mathfrak{C}_{0} \times \mathbb{C}^{*} \rightarrow\left(\mathbb{C}[z]_{\operatorname{deg}<d}\right)^{k-2}$ be defined by

$$
\mathcal{F}_{\mathfrak{q}}(\mathfrak{c}, \lambda):=\mathcal{F}(\mathfrak{c}, \mathfrak{q}, \lambda) .
$$

Corollary 3.3. Assume that $(\mathfrak{c}, \mathfrak{q}, \lambda)$ is defined over $\overline{\mathbb{Q}}$ with $\mathcal{F}(\mathfrak{c}, \mathfrak{q}, \lambda)=0$. Then, for almost every prime $p$, the derivative $D \mathcal{F}_{\mathfrak{q}}$ at $(\mathfrak{c}, \lambda)$ is invertible mod $p$. 
Proof. Since the point $(\mathfrak{c}, \mathfrak{q}, \lambda)$ is defined over $\overline{\mathbb{Q}}$, its coördinates may be written using algebraic integers, and reduced $\bmod p$. For all except finitely many values of $p$, the resulting reduction gives a genuine point, namely where the reductions of $(\mathfrak{c}, \mathfrak{q})$ are injective and the reduction of $f_{(\mathfrak{c}, \mathfrak{q}, \lambda)}$ has degree $d$. Since $D \mathcal{F}$ is invertible over $\overline{\mathbb{Q}}$, it may be written as $a / N$ for a matrix $a$ with algebraic integer entries and $N \in \mathbb{N}$; then the reduction modulo $p$ of $D \mathcal{F}$ is invertible for all primes not dividing $N$.

Before embarking in the proof of the Lemma, we first build up a description of the tangent space of Rat $_{d}$.

3.1. The tangent space to rational maps. Consider a rational map $f \in \operatorname{Rat}_{d}$. A tangent vector to $f$ is

$$
\dot{f}:=\left.\frac{\mathrm{d} f_{t}}{\mathrm{~d} t}\right|_{t=0}
$$

for a holomorphic family of rational maps $\left(f_{t}\right)_{|t|<\epsilon}$ with $f_{0}=f$.

For every $z \in \mathbb{P}^{1}(\mathbb{C})$, the vector $\dot{f}(z)$ is a tangent vector in $\mathbb{P}^{1}(\mathbb{C})$ at $f(z)$; in other words, $\dot{f}$ is a section of the pullback bundle $f^{*}\left(T \mathbb{P}^{1}(\mathbb{C})\right)$. It can be pulled back to a vector field on $\mathbb{P}^{1}(\mathbb{C})$, as

$$
\eta(z)=-\left(D_{z} f\right)^{-1}(\dot{f}(z))
$$

or, in coördinates, $\eta(z)=-\dot{f}(z) / f^{\prime}(z)$. Therefore, $\eta(z)$ is a meromorphic vector field on $\mathbb{P}^{1}(\mathbb{C})$, holomorphic away from critical points of $f$, and with a pole of order at most $m$ at critical points of multiplicity $m$.

Geometrically, $\eta(z)$ is the movement at time $t=0$ of the point $z_{t}=f_{t}^{-1}(f(z))$. This point $f_{t}$ can be followed away from critical points, by the Implicit Function Theorem.

We consider now local perturbations of $f$ at a critical point, i.e. we assume that the vector field $\dot{f}$ is given by a path $f_{t}=\phi_{t} \circ f \circ \psi_{t}^{-1}$ with $\phi_{t}, \psi_{t}$ analytic perturbations of the identity at the critical value and point $v, c$ respectively of $f$.

Let $c_{t}$ denote the critical point of $f_{t}$ and let $v_{t}$ denote its critical value; then $c_{t}=\psi_{t}(c)$ and $v_{t}=\phi_{t}(v)$. Let $\dot{c}$ denote the motion vector of $c_{t}$, and let $\dot{v}$ denote the motion vector of $v_{t}$. Then $\dot{c}=\dot{\psi}(c)$ and $\dot{v}=\dot{\phi}(v)$. Now $\dot{f}=\dot{\phi} \circ f-D f \circ \dot{\psi}$, because $\phi_{0}=\psi_{0}=$ id. Therefore,

$$
\eta+f^{*} \dot{\phi}=\dot{\psi}
$$

At $v$, the vector field $\dot{\phi}$ takes value $\dot{v}$, the vector field $\eta+f^{*} \dot{\phi}$ is holomorphic at $c$, and its constant term is $\dot{c}$. If $\dot{v}=0$, then $f^{*} \dot{\phi}$ is holomorphic near $c$ and vanishes at $c$.

Therefore, whenever we have a family of rational maps $\left(f_{t}\right)$ for which we can follow a critical point $c_{t}$ and its associated critical value $v_{t}$ with $\dot{v}=0$, the vector field $\eta$ is holomorphic near $c$ and coincides with $\dot{c}$ at $c$.

3.2. Proof of Lemma 3.2. For $i \in\{3, \ldots k\}$, we have

$$
f_{i}-f_{2}=\frac{F_{i}}{\lambda W_{1}}
$$

Recall that if $\mathcal{F}(\mathfrak{c}, \mathfrak{q}, \lambda)=0$, then $f_{i}=f_{2}$ for all $i \in\{3, \ldots, k\}$. We denote by $f$ this common rational map of degree $d$. If in addition $(\dot{\mathfrak{c}}, \dot{\mathfrak{q}}, \dot{\lambda})$ belongs to the Kernel 
of $D \mathcal{F}$ at $(\mathfrak{c}, \mathfrak{q}, \lambda)$, then

$$
\dot{f}_{i}-\dot{f}_{2}=\frac{\dot{F}_{i}}{\lambda W_{1}}-\frac{F_{i} \cdot\left(\dot{\lambda} W_{1}+\lambda \dot{W}_{1}\right)}{\left(\lambda W_{1}\right)^{2}}=0,
$$

so $\dot{f}_{i}=\dot{f}_{2}$ for all $i \in\{3, \ldots, k\}$. We denote by $\dot{f}$ this common tangent vector to $\mathrm{Rat}_{d}$ at $f$ and by $\eta$ the corresponding meromorphic vector field on $\mathbb{P}^{1}(\mathbb{C})$.

As $(\mathfrak{c}, \mathfrak{q}, \lambda)$ varies in $\mathfrak{Y}_{0} \times \mathbb{C}^{*}$, the $f_{i}$-preimages of the points $q_{1}=\infty$ and $q_{i}$ vary holomorphically: they are the points $c_{1, j}$ and $c_{i, j}$. According to the previous remark, we see that if $\dot{\mathfrak{q}}=0$ then, for all $i \in\{2, \ldots, k\}$, the meromorphic vector field $\eta$ is holomorphic near $c_{1, j}$, coincides with $\dot{c}_{1, j}$ at $c_{1, j}$, and furthermore is holomorphic near $c_{i, j}$ and coincides with $\dot{c}_{i, j}$ at $c_{i, j}$.

Thus, $\eta$ is a holomorphic vector field on the whole sphere $\mathbb{P}^{1}(\mathbb{C})$ and coincides with $\dot{c}_{i, j}$ at $c_{i, j}$. In particular, it vanishes at $c_{1,1}=\infty, c_{2,1}=0$ and $c_{3,1}=1$. A holomorphic vector field with at least 3 zeroes globally vanishes. Therefore, $\eta=0$ and $\dot{c}_{i, j}=0$ for all $(i, j)$. In addition, for all $i \in\{1, \ldots, k\}$, we have that $\dot{W}_{i}=0$ and for all $i \in\{3, \ldots k\}$, we have that $0=\dot{F}_{i}=\dot{\lambda} q_{i} W_{1}$. This shows that $\dot{\lambda}=0$.

Let us summarize: if $\mathcal{F}(\mathfrak{c}, \mathfrak{q}, \lambda)=0$ and if $(\dot{\mathfrak{c}}, 0, \dot{\lambda})$ belongs to the Kernel of $D \mathcal{F}$ at $(\mathfrak{c}, \mathfrak{q}, \lambda)$, then $\dot{\mathfrak{c}}=0$ and $\dot{\lambda}=0$. So, the restriction of $D_{(\mathfrak{c}, \mathfrak{q}, \lambda)} \mathcal{F}$ to $T_{\mathfrak{c}} \mathfrak{C}_{0} \times\{0\} \times T_{\lambda} \mathbb{C}$ is injective. Since $T_{\mathfrak{c}} \mathfrak{C}_{0} \times\{0\} \times T_{\lambda} \mathbb{C}$ and $T_{0}\left(\mathbb{C}[z]_{\operatorname{deg}<d}\right)^{k-2}$ have the same dimension, that is $(k-2) d$, this restriction is an isomorphism as required.

\section{Finding a SOlution in a Finite Field}

We describe in this section an efficient method of finding a rational function over a finite field with prescribed critical values and multiplicities.

We start by recalling some facts about univariate polynomials over non-algebraicallyclosed fields $\mathbb{k}$ of arbitrary characteristic. For this we need some notation:

Notation 4.1. An ordered sequence $\alpha=\left(\alpha_{1}, \ldots, \alpha_{k}\right)$ with $\alpha_{1} \geq \cdots \geq \alpha_{k}>0$ and $\sum \alpha_{i}=d$ is called a partition of $d$. With the shorthand notation

$$
\beta^{\mu}:=(\underbrace{\beta, \ldots, \beta}_{\mu \text { times }})
$$

we can always write $\alpha=\left(\alpha_{1}, \ldots, \alpha_{k}\right)=\left(\beta_{1}^{\mu_{1}}, \ldots, \beta_{n}^{\mu_{n}}\right)$ with $\beta_{1}>\cdots>\beta_{n}$ and appropriate $\mu_{i}$. For example, $13=4+3+2+2+2$ is written as $\alpha=(4,3,2,2,2)=$ $\left(4^{1}, 3^{1}, 2^{3}\right)$.

The partition $\alpha^{*}$ defined by $\alpha_{j}^{*}:=\#\left\{i: \alpha_{i} \geq j\right\}$ is called the dual partition of $\alpha$. For example, $\alpha^{*}=(5,5,2,1)$.

Let $f \in \mathbb{k}[x]$ be a degree- $d$ polynomial, let $l_{i}=\left(x-C_{i}\right) \in \overline{\mathbb{k}}[x]$ be its distinct linear factors over an algebraic closure of $\mathbb{k}$, and let $\alpha_{i}$ be their multiplicities, so that

$$
f=\prod_{i=1}^{k} l_{i}^{\alpha_{i}} .
$$

Without restriction we can assume $\alpha_{1} \geq \cdots \geq \alpha_{k}$ and $\alpha=\left(\alpha_{1}, \ldots, \alpha_{k}\right)$ is a partition of $d$. In this situation we say that $f$ is of shape $\alpha$.

If we write $\alpha=\left(\alpha_{1}, \ldots, \alpha_{k}\right)=\left(\beta_{1}^{\mu_{1}}, \ldots, \beta_{n}^{\mu_{n}}\right)$ as above, we can write

$$
f=\prod_{i=1}^{n} f_{i}^{\beta_{i}}
$$


with $\operatorname{deg} f_{i}=\mu_{i}$ and $f_{i}$ the product of those linear forms that have multiplicity $\beta_{i}$. In this situation the $f_{i}$ are coprime.

Lemma 4.2. Let $\mathbb{k}$ be a field of characteristic $p$, let $f \in \mathbb{k}[x]$ be a univariate polynomial of shape $\alpha$ and write $f=\prod_{i=1}^{k} l_{i}^{\alpha_{i}}$ with $l_{i} \in \overline{\mathbb{k}}[x]$. If $\alpha_{i}<p$ for all $i$ then

$$
\operatorname{gcd}\left(f, f^{\prime}\right)=\prod_{i=1}^{k} l_{i}^{\alpha_{i}-1}
$$

Proof. We have

$$
f^{\prime}=\sum_{i=1}^{k} \alpha_{i} \frac{f}{l_{i}} l_{i}^{\prime}=\left(\prod l_{i}^{\alpha_{i}-1}\right) \sum_{i=1}^{k} \alpha_{i}\left(\prod_{j \neq i} l_{j}\right) l_{i}^{\prime} .
$$

This shows that $\prod_{i=1}^{k} l_{i}^{\alpha_{i}-1}$ divides the gcd. Assume now that there is another linear factor $l$ in the gcd. Since the gcd divides $f$ there exists an index $s$ with $l=l_{s}$. Since the gcd divides $f^{\prime}$ we have that $l$ divides

$$
\sum_{i=1}^{k} \alpha_{i}\left(\prod_{j \neq i} l_{j}\right) l_{i}^{\prime}
$$

Now all summands except for $\alpha_{s}\left(\prod_{j \neq s} l_{j}\right) l_{s}^{\prime}$ are divisible by $l$. Since $\alpha_{s}$ and $l_{s}^{\prime}$ are nonzero in $\mathbb{k}$ and $\overline{\mathbb{k}}[x]$ respectively, it follows that $l$ must divide $\prod_{j \neq i} l_{j}$. This is impossible since the $l_{s}$ are pairwise coprime.

Corollary 4.3. With the notations of the previous Lemma we have

$$
\operatorname{gcd}\left(f, f^{\prime}, \ldots, f^{(e)}\right)=\prod_{i: \alpha_{i}>e} l_{i}^{\alpha_{i}-e} .
$$

Proof. Lemma 4.2 and induction.

Corollary 4.4. With the notations above let $\alpha^{*}$ be the dual partition of $\alpha$. Then

$$
\alpha_{e}^{*}=\operatorname{deg} \operatorname{gcd}\left(f, f^{\prime}, \ldots, f^{(e-1)}\right)-\operatorname{deg} \operatorname{gcd}\left(f, f^{\prime}, \ldots, f^{(e)}\right) .
$$

Proof. We have

$$
g_{e}:=\frac{\operatorname{gcd}\left(f, f^{\prime}, \ldots, f^{e-1}\right)}{\operatorname{gcd}\left(f, f^{\prime}, \ldots, f^{e}\right)}=\frac{\prod_{i: \alpha_{i}>e-1} l_{i}^{\alpha_{i}-e+1}}{\prod_{i: \alpha_{i}>e^{2}} l_{i}^{\alpha_{i}-e}}=\prod_{i: \alpha_{i} \geq e} l_{i} .
$$

It follows that

$$
\operatorname{deg} g_{e}=\operatorname{deg} \prod_{i: \alpha_{i} \geq e} l_{i}=\#\left\{i: \alpha_{i} \geq e\right\}=\alpha_{e}^{*} .
$$

Algorithm 4.5 (Compute the shape of a polynomial).

Given: a polynomial $f \in \mathbb{k}[x]$

Return: the shape $\left(\alpha_{1}, \ldots, \alpha_{k}\right)$ of $f$.

Write $d=\operatorname{deg}(f)$. For each $e=0, \ldots, d$, compute $g_{e}=\operatorname{gcd}\left(f, f^{\prime}, \ldots, f^{(e)}\right)$. For each $e=1, \ldots, d$ define then $\alpha_{e}^{*}=\operatorname{deg}\left(g_{e-1}\right)-\operatorname{deg}\left(g_{e}\right)$. Return the dual of the partition $\left(\alpha_{1}^{*}, \ldots, \alpha_{d}^{*}\right)$.

Proof of validity. This directly follows from Corollary 4.4 
We may collect linear factors of the same multiplicity, so as to avoid field extensions:

Corollary 4.6. With the notation of Lemma 4.2 choose $\beta_{1}>\cdots>\beta_{n}$ among the $\alpha_{i}$ such that $f=\prod_{i=1}^{n} f_{i}^{\beta_{i}}$ with $f_{j}=\prod_{i: \alpha_{i}=\beta_{j}} l_{i}$. Then the $f_{i}$ are defined over $\mathbb{k}$.

Proof. Since the calculation of a gcd does not require field extensions, we have

$$
\operatorname{gcd}\left(f, f^{\prime}, \ldots, f^{(e)}\right) \in \mathbb{k}[x] .
$$

By Corollary 4.3 we then have

So

$$
g_{j}:=\frac{\operatorname{gcd}\left(f, f^{\prime}, \ldots, f^{\left(\beta_{j}-1\right)}\right)}{\operatorname{gcd}\left(f, f^{\prime}, \ldots, f^{\left(\beta_{j}\right)}\right)}=\frac{\prod_{i: \beta_{i}>\beta_{j}-1} f_{i}^{\beta_{i}-\beta_{j}+1}}{\prod_{i: \beta_{i}>\beta_{j}} f_{i}^{\beta_{i}-\beta_{j}}}=\prod_{i=1}^{j} f_{i} \in \mathbb{k}[x]
$$

$$
f_{j}=\frac{g_{j}}{g_{j+1}} \in \overline{\mathbb{k}}[x] \cap \mathbb{k}(x)=\mathbb{k}[x] .
$$

We are now ready to describe our algorithm searching for rational maps over $\mathbb{F}_{p}$.

Algorithm 4.7 (Compute all rational maps over $\mathbb{k}$ with given shape above given points).

Given: a finite field $\mathbb{k}$, a list of points $\bar{Q}_{1}, \ldots, \bar{Q}_{k} \in \mathbb{P}^{1}(\mathbb{k})$, an integer $d$, and a list of partitions $\left(\alpha_{1}, \ldots, \alpha_{k}\right)$ of $d$ with $\alpha_{i}=\left(\alpha_{i, 1}, \ldots, \alpha_{i, \ell_{i}}\right)$ satisfying $\sum_{i} \sum_{j}\left(\alpha_{i, j}-1\right)=$ $2 d-2$

Return: all rational maps over $\mathbb{k}$ of degree $d$ such that every $\bar{Q}_{i}$ has $\ell_{i}$ preimages with local degrees $\alpha_{i, 1}, \ldots, \alpha_{i, \ell_{i}}$ respectively.

We choose a Möbius transformation $M \in \mathrm{PSL}_{2}(\mathbb{k})$ sending $\bar{Q}_{1}$ to $\infty$ and $\bar{Q}_{2}$ to 0 .

We write each partition $\alpha_{i}$ in compacted form as $\alpha_{i}=\left(\beta_{i, 1}^{\mu_{i, 1}}, \ldots, \beta_{i, n_{i}}^{\mu_{i, n_{i}}}\right)$.

We enumerate all $\ell_{1}$-tuples of monic polynomials $\left(f_{1}, \ldots, f_{n_{1}}\right)$ with $\operatorname{deg}\left(f_{j}\right)=$ $\mu_{i, j}$, and all $\ell_{2}$-tuples of monic polynomials $\left(g_{1}, \ldots, g_{n_{2}}\right)$ with $\operatorname{deg}\left(g_{j}\right)=\mu_{2, j}$.

For each such pair of tuples, we compute

$$
W_{1}=\prod_{j=1}^{n_{1}} f_{j}^{\beta_{1, j}} \text { and } W_{2}=\prod_{j=1}^{n_{2}} g_{j}^{\beta_{2, j}} .
$$

Using Algorithm 4.5, we filter those $\left(W_{1}, W_{2}\right)$ such that the shape of $W_{1}$ is $\alpha_{1}$ and the shape of $W_{2}$ is $\alpha_{2}$ (this fails only if a pair $f_{i}, g_{j}$ is not coprime).

By computing their g.c.d., we filter those $\left(W_{1}, W_{2}\right)$ such that $W_{1}$ and $W_{2}$ are coprime.

For each $i=3, \ldots, k$, let $\Lambda_{i} \subset \mathbb{k}$ be the set of $\lambda \in \mathbb{k}$ such that the shape (computed using Algorithm 4.5) of $W_{i}:=W_{2}-\lambda M\left(\bar{Q}_{i}\right) W_{1}$ is $\alpha_{i}$. We filter those rational maps for which $\bigcap_{i=3}^{k} \Lambda_{i}$ is non-empty.

We return all the rational maps $M^{-1} \circ\left(W_{2} / \lambda W_{1}\right)$, for all $\lambda \in \bigcap_{i=3}^{k} \Lambda_{i}$, that survived the filtering.

Proof of validity. Let first $f:=M^{-1} \circ\left(W_{2} / \lambda W_{1}\right)$ be a rational map returned by the algorithm. For $i=2, \ldots, k$, consider the rational map $f_{i}=M^{-1} \circ\left(W_{i} / \lambda W_{1}\right)$. By the very definition of $W_{i}$ (compare with (3p), we have $f=f_{2}=\cdots=f_{k}$. On the other hand, the $f_{i}$-preimages of $\bar{Q}_{i}$ are the zeroes of $W_{i}$, so they have multiplicities $\alpha_{i}$. 
On the other hand, let $f$ be a rational map such that every $\bar{Q}_{i}$ has $\ell_{i}$ preimages with local degrees $\alpha_{i, 1}, \ldots, \alpha_{i, \ell_{i}}$ respectively. Then, for every Möbius transformation $M$ sending $\bar{Q}_{1}$ to $\infty$ and $\bar{Q}_{2}$ to 0 , the rational map $M \circ f$ will be of the form $W_{2} / \lambda W_{1}$, for monic polynomials $W_{1}, W_{2}$ of respective shapes $\alpha_{1}, \alpha_{2}$ and a scalar $\lambda \in \mathbb{k}$. Furthermore, by Corollary 4.6 , both $W_{1}$ and $W_{2}$ factor over $\mathbb{k}[x]$ into polynomials of degrees $\mu_{1, j}$ and $\mu_{2, j}$ respectively. The rational map $M \circ f-\bar{Q}_{i}$ will be of the form $W_{i} / \lambda W_{1}$ with $W_{i}$ of shape $\alpha_{i}$; therefore, that solution $f$ will be returned by the algorithm.

Remark 4.8. Algorithm 4.7 is the most computationally-intensive part of our procedure. Its performance is improved in the following ways:

(1) If $\mu_{i, j}=1$ for some $i, j$, then we may assume, after permuting the shapes $\alpha_{i}$, that $i=1$ so that $W_{1}$ contains a power of a linear factor $f_{i}^{\beta_{i, j}}$. Fixing the corresponding preimage of $\infty$ to be $\infty$ amounts to the choice $f_{i}=1$, so that the degree of $W_{1}$ is actually $d-\beta_{i, j}$. This speeds up the search by a factor $p$.

Similarly, if up to permutation of the indices there are more $\mu_{i, j}=1$, with $i \in\{1,2\}$, then the corresponding factors may be assumed to be $x$ and $x-1$.

On the other hand, if all $\mu_{i, j} \geq 2$, then no normalization of the critical points may be assumed, and in particular $\infty$ should not be assumed to be a preimage of some $\bar{Q}_{i}$.

(2) When using Corollary 4.4 one can detect a wrong shape already if $\operatorname{gcd}\left(f, f^{\prime}\right)$ or for that matter any $\operatorname{gcd}\left(f, \ldots, f^{(e)}\right)$ with $e<\alpha_{1}$ has the wrong degree. We stop the calculation of gcd's as soon as this happens. This speeds up the process by a factor of about $\alpha_{1}$. Similarly, as soon as the intersection of the $\Lambda_{i}$ already computed is empty, the pair $\left(W_{1}, W_{2}\right)$ should be discarded.

(3) If the largest $\mu_{i, j}$ is small enough (e.g. 7 or 8 in $\mathbb{F}_{11}$ ) we can enumerate all monic irreducible homogeneous polynomials of degree $\leq \mu_{i, j}$ and build the $f_{i}$ and $g_{i}$ out of them, while taking care that no irreducible piece is used twice. We can then omit checking shape and coprimeness of $W_{1}$ and $W_{2}$ as these conditions are then automatically satisfied.

Example 4.9. Over $\mathbb{F}_{11}$ we searched for a rational map of shape $(4,3,2,2,2)$, $(4,3,2,2,2)$ and $(4,3,2,2,2)$; we chose $\bar{Q}_{1}=\infty$ and $\bar{Q}_{2}=0$, and didn't specify $\bar{Q}_{3}$, letting on the contrary the algorithm determine choose it for us. We found the solution

$$
\frac{W_{2}}{W_{1}}=\frac{x^{4}(x+3)^{3}\left(x^{3}-3 x-5\right)^{2}}{(x-5)^{3}\left(x^{3}+3 x^{2}+2 x+3\right)^{2}} .
$$

It has indeed the desired shape as we have the following factorisation

$$
W_{2}+4 W_{1}=(x-1)^{4}(x-3)^{3}\left(x^{3}-2 x-3\right)^{2},
$$

which implies, for the choice $\bar{Q}_{3}=1$, the value $\lambda=-4$.

\section{Lifting A SOLUtion FROM $\mathbb{F}_{p}$ TO $\mathbb{Z}_{p}$}

The lift from $\mathbb{F}_{p}$ to $\mathbb{Z}_{p}$ is done using Hensel's lemma (namely, Newton's method in positive characteristic): 
Proposition 5.1 (Hensel's Lemma). Let $\mathcal{F}=\left(F_{1}, \ldots, F_{m}\right)$ be a vector of polynomials, with $F_{i} \in \mathbb{Z}\left[x_{1}, \ldots, x_{m}\right]$, and let $J=\left(\frac{\mathrm{d} F_{i}}{\mathrm{~d} x_{j}}\right)$ be the Jacobian matrix of $F$. Assume that $a=\left(a_{1}, \ldots, a_{m}\right) \in \mathbb{Z}^{m}$ satisfies

$$
\mathcal{F}(a) \equiv 0 \quad \bmod p^{N}
$$

that $J(a)$ is invertible modulo $p^{N}$, and let $J^{-1}(a)$ be an inverse modulo $p^{N}$. Then

$$
\mathcal{F}\left(a+b p^{N}\right) \equiv 0 \quad \bmod p^{2 N},
$$

for

$$
b:=-\frac{F(a)}{p^{N}} J^{-1}(a) .
$$

Furthermore $J\left(a+b p^{N}\right)$ is invertible modulo $p^{2 N}$.

Proof. $F(a)$ is divisible by $p^{N}$ since $F(a) \equiv 0 \bmod p^{N}$. Therefore $b$ is well defined. We have

$$
\begin{aligned}
F\left(a+p^{N} b\right) & \equiv F(a)+b J(a) p^{N} \bmod p^{2 N} \\
& \equiv F(a)-\frac{F(a)}{p^{N}} J^{-1}(a) J(a) p^{N} \bmod p^{2 N} \\
& \equiv 0 \quad \bmod p^{2 N} .
\end{aligned}
$$

The invertibility holds more generally. Let $A$ and $B$ be matrices with $A B \equiv \mathbb{1}$ $\bmod p^{N}$. We can then write

$$
A B \equiv \mathbb{1}+p^{N} C \quad \bmod p^{2 N} .
$$

In this situation we have

$$
\begin{aligned}
A\left(B-p^{N} B C\right) & \equiv \mathbb{1}+p^{N} C-p^{N} A B C \quad \bmod p^{2 N} \\
& \equiv \mathbb{1} \quad \bmod p^{2 N}
\end{aligned}
$$

since $A B \equiv \mathbb{1} \bmod p^{N} ;$ so $B^{\prime}=B-p^{N} B C$ is an inverse to $A$ modulo $p^{2 N}$.

Consider the following data: a ring $\mathbb{k}$; a family of polynomials $W_{i} \in \mathbb{k}[x]$ of degree at most $d$, for $i=1, \ldots, k$, with factorisations $W_{i}=\prod_{j=1}^{n_{j}} W_{i, j}^{\beta_{i, j}}$; a parameter $\lambda \in \mathbb{k}^{\times}$; and a sequence of points $Q_{1}=\infty, Q_{2}, \ldots, Q_{k} \in \mathbb{P}^{1}(\mathbb{k})$. We say that they are coherent if $W_{i} / \lambda W_{1}+Q_{i}$ is independent of $i=2, \ldots, k$.

We say that they are normalised if the following holds: the first three values $Q_{1}, Q_{2}, Q_{3}$ are $\infty, 0,1$ respectively; and the first three preimages $C_{1,1}, C_{2,1}, C_{3,1}$ are also respectively $\infty, 0,1$. This means that we assume that $W_{1}$ has degree $d-\alpha_{1,1}$, that $W_{2,1}=x^{\alpha_{2,1}}$, and that $W_{3,1}=(x-1)^{\alpha_{3,1}}$.

Note that this assumption is not innocuous: it may well be that no critical point $C_{i, j}$ is defined over $\mathbb{k}$. The normalization may be imposed at no cost if (after permutation of the indices) $\mu_{1,1}=\mu_{2,1}=\mu_{3,1}=1$.

We are now ready to detail the lifting algorithm. Out of coherent data in $\mathbb{F}_{p}$ and a parameter $N$, it computes a $p^{-N}$-approximation of the corresponding coherent data in $\mathbb{Z}_{p}$, in the form of an approximation in $\mathbb{Z} / p^{N}$.

Algorithm 5.2 (Lift a solution $p$-adically).

Given: coherent data $\bar{W}_{i}=\prod_{j=1}^{n_{j}} \bar{W}_{i, j}^{\beta_{i, j}} \in \mathbb{F}_{p}[x], \bar{\lambda} \in \mathbb{F}_{p}^{\times}$, and $\bar{Q}_{i} \in \mathbb{P}^{1}\left(\mathbb{F}_{p}\right)$; a parameter $N \in \mathbb{N}$; and lifts $Q_{i} \in \mathbb{P}^{1}\left(\mathbb{Z} / p^{N}\right)$ of the points $\bar{Q}_{i}$ 


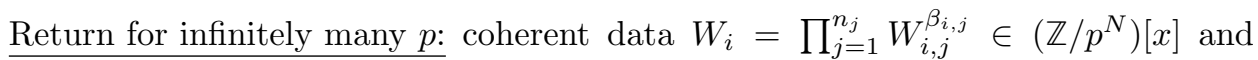
$\lambda \in\left(\mathbb{Z} / p^{N}\right)^{\times}$that reduce $\bmod p$ to $\bar{W}_{i}$.

First, we assume that the data may be normalised. This amounts to requiring at least three of the $W_{i, j}$, for distinct $i$ 's, to have a linear factor. This holds for a positive proportion of primes $p$. If no such three factors exist, the algorithm aborts. Otherwise, we silently replace the three corresponding $\beta_{i, j}^{\mu_{i, j}}$ by $\left(\beta_{i, j}^{1}, \beta_{i, j}^{\mu_{i, j}-1}\right)$ in the shapes so as to create a term with $\mu_{i, j}=1$.

We write now each $W_{i, j}$ in the form

$$
W_{i, j}=x^{\mu_{i, j}}+\sum_{s=1}^{\mu_{i, j}} w_{i, j, s} x^{\mu_{i, j}-s},
$$

for unknowns $w_{i, j, s} \in \mathbb{Z} / p^{N}$.

Recall from (3) the expressions $F_{i}=W_{i}+\lambda q_{i} W_{1}-W_{2}$ and $\mathcal{F}=\left(F_{3}, \ldots, F_{k}\right)$. The $F_{i}$ are polynomials in the variables $\left\{w_{i, j, s}:(i, j) \neq(1,1),(2,1),(3,1)\right\} \cup\{\lambda\}$. We lift the coëfficients of the coherent data $\left(\bar{W}_{i}, \bar{\lambda}\right)$ to $\mathbb{Z}$, to obtain an initial parameter $a^{0}=$ $\left(w_{1,2,1}^{0}, \ldots, w_{k, n_{k}, \mu_{k, n_{k}}}^{0}, \lambda^{0}\right)$. Since the original data is coherent, we have $\mathcal{F}\left(a^{0}\right) \equiv 0$ $(\bmod p)$. For almost all $p$, the Jacobian $D \mathcal{F}$ is invertible by Corollary 3.3 if $D \mathcal{F}$ is not invertible at $a^{0}$, then we abort the algorithm. Otherwise, we apply repeatedly Hensel's Lemma 5.1 to obtain a solution $a$ to $\mathcal{F}(a) \equiv 0\left(\bmod p^{N}\right)$.

Finally, we reconstruct the polynomials $W_{i, j}$ out of their coëfficients (which are just coördinates of $a$ ).

Proof of validity. The invertibility of the Jacobian was expressed in Corollary 3.3 in terms of the variables $C_{i, j}$. This does not make any difference: here we express them in terms of the $w_{i, j, s}$, which are elementary symmetric functions of the $C_{i, j}$.

Example 5.3. Consider the shapes $\alpha_{1}=\alpha_{2}=\alpha_{3}=\left(4^{1}, 3^{1}, 2^{3}\right)$. Our example

$$
\begin{aligned}
W_{1} & =(x-5)^{3}\left(x^{3}+3 x^{2}+2 x+3\right)^{2} \\
W_{2} & =x^{4}(x+3)^{3}\left(x^{3}-3 x-5\right)^{2} \\
W_{3}=W_{2}+4 W_{1} & =(x-1)^{4}(x-3)^{3}\left(x^{3}-2 x-3\right)^{2}
\end{aligned}
$$

gives a vector of coëfficients

$$
\begin{aligned}
a^{0}= & \left(w_{1,2,1}, w_{1,3,1}, w_{1,3,2}, w_{1,3,3},\right. \\
& w_{2,2,1}, w_{2,3,1}, w_{2,3,2}, w_{2,3,3} \\
& \left.w_{3,2,1}, w_{3,3,1}, w_{3,3,2}, w_{3,3,3}, \lambda\right) \\
= & (-5,3,2,3,3,-3,0,-5,-3,-2,0,-3,-4)
\end{aligned}
$$

with $F\left(w_{0}\right) \equiv 0(\bmod 11)$. The lift is

$$
a^{1}=(50,-41,13,25,-19,-33,19,-60,-47,11,-46,-58,51)
$$

with $F\left(w_{1}\right) \equiv 0\left(\bmod 11^{2}\right)$. We can continue this process inductively. Notice that the precision doubles in every step.

\section{Promoting a solution from $\mathbb{Z}_{p}$ to A number field $\mathbb{K}$}

If the Hurwitz problem has a solution over $\mathbb{Z}$ that reduces to a given solution over $\mathbb{F}_{p}$, then Hensel lifting will find it after a finite number of steps. Unfortunately the solutions usually involve fractional coëfficients, and are usually defined over a finite extension $\mathbb{K}$ of $\mathbb{Q}$. Our first goal will therefore be to determine this extension. 
Consider a degree- $e$ extension $\mathbb{K}$ of the rationals, and $a \in \mathbb{K}$. Then $1, a, \ldots, a^{e}$ are linearly dependent over $\mathbb{Q}$, and therefore also over $\mathbb{Z}$, i.e there exists a polynomial

$$
P=p_{0}+p_{1} t+\cdots+p_{e} t^{e}
$$

with all $p_{i} \in \mathbb{Z}$ and $P(a)=0$. Let now $p \in \mathbb{N}$ be a prime such that $P$ splits over $\mathbb{Z}_{p}$; so that we may view $\mathbb{Q}(a)$ as a subfield of $\mathbb{Q}_{p}$. Assume also that $a$ is invertible modulo $p$, so that we may consider $a$ as an element of $\mathbb{Z}_{p}$.

Consider now $\tilde{a} \equiv a\left(\bmod p^{N}\right)$; then we have the equation

$$
P(\tilde{a})=p_{e+1} p^{N}
$$

which is linear in $\left\{p_{0}, \ldots, p_{e+1}\right\}$. We use the LLL algorithm 13 to find small integer solutions to this linear equation. The default implementation uses a simple heuristic to guess the correct precision $N$ and the correct extension degree: for a initial precision we start with extension degree $e=1$ and increase $e$ until a solution is found (i.e. $F(\tilde{a})=0 \bmod p^{2 N}$ ) or the computed shortest lattice basis vector norm is the same for $e$ and $e-1$. If the computed vector norm did not change, we increase the $p$-adic precision. If we have a-priori knowledge about the minimum or maximum expected extension degree, then it can be passed to the algorithm, which is more likely to find quickly a solution.

The following algorithm is described as a process that, receiving as input an infinite feed of ever-more-precise approximations of a $p$-adic number that is known to be algebraic, produces an infinite stream of ever-more-likely minimal polynomials of that $p$-adic number.

Algorithm 6.1 (Convert a $p$-adic number to an algebraic number). Given: approximations, to arbitrary precision, of an algebraic number $a \in \mathbb{Z}_{p}$

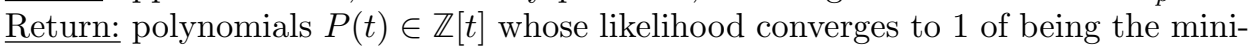
mal polynomial of $a$, as the precision of $a$ improves.

Assume that, for each $N \in \mathbb{N}$, the algorithm may receive an approximation $a_{N}$, to $N$ base- $p$ digits, of $a$. The element $a_{N}$ is represented as an integer in $\left\{0, \ldots, p^{N}-1\right\}$.

Start with $d=1$ and $N=1$. Then, repeat the following. Consider the lattice in $\mathbb{R}^{d+1}$ generated by the columns of the matrix

$$
M=\left(\begin{array}{ccccc}
p^{N} & -a_{N} & -a_{N}^{2} & \ldots & -a_{N}^{d} \\
0 & 1 & 0 & \ldots & 0 \\
0 & 0 & 1 & \ldots & 0 \\
\vdots & \vdots & & \ddots & \vdots \\
0 & 0 & 0 & \ldots & 1
\end{array}\right) .
$$

Using the LLL algorithm, find a vector $\left(P_{0}, P_{1}, \ldots, P_{d}\right)$ in the lattice, of small norm $\theta_{N, d}$. Form the polynomial $P(t)=\sum_{i=0}^{d} P_{i} t^{i}$.

If $P\left(a_{N}\right) \equiv 0\left(\bmod p^{2 N}\right)$, or if $d>1$ and $\theta_{N, d}=\theta_{N, d-1}$, then output $P$ as a candidate polynomial. Repeat then, after having incremented $d$ if the first case holds, and doubled $N$ otherwise.

Proof of validity. The algorithm repeatedly increases $N$ and $d$. Note that the polynomials returned may have degree $<d$, so increasing $d$ is harmless, and the precision is increased as soon as increase in maximal degree does not improve the solution.

Let $\left(P_{0}, \ldots, P_{d}\right)$ be a short lattice vector. Then this vector is

$$
M \cdot{ }^{t}\left(P\left(a_{N}\right) / p^{N}, P_{1}, \ldots, P_{d}\right)
$$


and in particular $P\left(a_{N}\right) \equiv 0\left(\bmod p^{N}\right)$. On the other hand, the coëfficients $P_{i}$ are small, so $P$ is likely to be the minimal polynomial of $a$.

Example 6.2. Considering our example $\alpha_{1}=\alpha_{2}=\alpha_{3}=\left(4^{1}, 3^{1}, 2^{3}\right)$ we found

$w_{1,2,1}=1400834756308742009361916361765119584358776523123371526525883115012 \in \mathbb{Z} / 11^{2^{6}}$

and $P_{1,2,1}\left(w_{1,2,1}\right) \equiv 0\left(\bmod 11^{2^{6}}\right)$ for

$$
P_{1,2,1}(t)=39 t^{6}+117 t^{5}+195 t^{4}+195 t^{3}+141 t^{2}+63 t+16 .
$$

Higher precision values of $w_{1,2,1}$ are also zeroes of the same polynomial $P_{1,2,1}$. We take this as a hint that $P_{1,2,1}$ is indeed the minimal polynomial of the coördinate $w_{1,2,1}$ in the lift of our finite field solution.

Having found the minimal polynomials $P_{i, j, s} \in \mathbb{Z}[t]$ for all coördinates $w_{i, j, s}$ of our solution vector, we determine the field $\mathbb{K}$ on which they are all defined, as the compositum of all field extensions defined by the $P_{i, j, s}$. If these field extensions were independent, then we should just consider all zeroes in $\mathbb{C}$ of the $P_{i, j, s}$ and return the corresponding rational functions.

However, in general, the field extensions will be highly dependent. To simplify notation, let us assume that all $P_{i, j, s}$ are of degree $e$, and that $\mathbb{K}$ itself is a degree$e$ extension. Then there are $e$ possible values for each coördinate. At this stage we do not know how to combine these single coördinate solutions to a solution vector (there are $e^{d(k-2)}$ possible combinations). To solve this problem we use the following method.

To illustrate our method, consider $a, b \in \mathbb{K}$ and let $P_{a}, P_{b}, P_{a+b} \in \mathbb{Z}[x]$ be minimal polynomials of $a, b, a+b$ respectively. Assume furthermore that $P_{a}, P_{b}$ and $P_{a+b}$ are of degree $e$, and let $a_{1}, \ldots a_{e}, b_{1}, \ldots, b_{e}$ and $c_{1}, \ldots, c_{e}$ respectively be approximations over $\mathbb{C}$ of the zeroes of $P_{a}, P_{b}$ and $P_{a+b}$. Consider the $e \times e$ "root compatibility matrix" $M=\left(M_{i, j}\right)$ defined by

$$
M_{i, j}= \begin{cases}1 & \text { if there exists } k \text { with } a_{i}+b_{j} \approx c_{k} \\ 0 & \text { otherwise }\end{cases}
$$

If $M$ is a permutation matrix, then it describes which root $b_{j}$ should be paired with $a_{i}$, namely it is characterised by $M_{i, j}=1$. In this manner, all other coördinates are chosen, dependent on the first choice of a root of $P_{1,2,1}$.

Example 6.3. Tentative minimal polynomials of $w_{1,3,1}$ and $a=w_{1,2,1}+w_{1,3,1}$ are

$$
\begin{aligned}
P_{1,3,1} & =28431 t^{6}+255879 t^{5}+982449 t^{4}+2056509 t^{3}+2465721 t^{2}+1597239 t+439138 \\
P_{a} & =28431 t^{6}+341172 t^{5}+1844856 t^{4}+5660928 t^{3}+10384524 t^{2}+10807344 t+5068144
\end{aligned}
$$

We obtain the following approximate zeroes over $\mathbb{C}$ using Brent's method, implemented in PARI 2]; we preserve the ordering in which the roots were returned.

$$
\begin{array}{ccc}
w_{1,2,1} & w_{1,3,1} & w_{1,2,1}+w_{1,3,1} \\
\hline-.150+.807 i & -1.5-1.02 i & -2.161-1.184 i \\
-.150-.807 i & -1.5+1.02 i & -2.162+1.184 i \\
-.5+.440 i & -2.012-.377 i & -2-1.462 i \\
-.5-.440 i & -2.012+.377 i & -2+1.462 i \\
-.850+.807 i & -.988-.377 i & -1.839-1.184 i \\
-.850-.807 i & -.988+.377 i & -1.839+1.184 i
\end{array}
$$


Now consider the compatibility matrix $M$. It is

$$
\left(\begin{array}{llllll}
0 & 0 & 0 & 1 & 0 & 0 \\
0 & 0 & 1 & 0 & 0 & 0 \\
0 & 1 & 0 & 0 & 0 & 0 \\
1 & 0 & 0 & 0 & 0 & 0 \\
0 & 0 & 0 & 0 & 0 & 1 \\
0 & 0 & 0 & 0 & 1 & 0
\end{array}\right),
$$

i.e. $M_{i, j}$ is 1 precisely when $\left(w_{1,2,1}\right)_{i}+\left(w_{1,3,1}\right)_{j}$ approximates one of the values in the last column of the table above. We note that $M$ is a permutation matrix. Applying the permutation $M$ to the list of roots $\left(w_{1,3,1}\right)_{i}$ of $P_{1,3,1}$ leads to a valid coördinate pairing: now $\left(M w_{1,3,1}\right)_{i}$ corresponds to the $\left(w_{1,2,1}\right)_{i}$.

Example 6.4. There are situations in which the matrix $M$ is not a permutation matrix but nevertheless contains useful information. Consider the finite algebraic set

$$
S=\{(i, \sqrt{2}),(-i, \sqrt{2}),(i,-\sqrt{2}),(-i,-\sqrt{2})\} .
$$

In this case the minimal polynomials of the coördinates are $x^{2}+1$ and $y^{2}-2$ and the root compatibility matrix is $M=\left(\begin{array}{ll}1 & 1 \\ 1 & 1\end{array}\right)$. All 4 possible pairings lead to correct solutions.

There are also situations in which the matrix $M$ differs from the permutation matrix giving the correct identification of coördinates, even with exact arithmetic. For example, if $\xi$ denotes a fifth root of unity, and

$$
S=\left\{\left(\xi, \xi^{2}\right),\left(\xi^{2}, \xi^{4}\right),\left(\xi^{3}, \xi\right),\left(\xi^{4}, \xi^{3}\right)\right\}
$$

then the coördinates have respectively $x^{4}+x^{3}+x^{2}+x+1$ and $y^{4}+y^{3}+y^{2}+y+1$ as their minimal polynomial, and the root compatibility matrix is insufficient to recover $S$. Indeed the sum of the coördinates $z=x+y$, which has minimal polynomial $z^{4}+2 z^{3}+4 z^{2}+3 z+1$, does not distinguish solutions in $S$ from the non-solutions

$$
S^{\prime}=\left\{\left(\xi, \xi^{3}\right),\left(\xi^{2}, \xi\right),\left(\xi^{3}, \xi^{4}\right),\left(\xi^{4}, \xi^{2}\right)\right\}
$$

Note that $S$ and $S^{\prime}$ are distinguished by the equation $x^{2}-y$ which holds in $S$ but not in $S^{\prime}$. A linear form $a x+b y$ with $0 \neq a \neq b \neq 0$ would also do.

There are also situations with more than two variables in which all compatibility matrices between two variables lead to possible pairings, but their combined information is not enough to identify the correct solutions. For example, consider

$$
S=\left\{\left(\varepsilon_{1} \sqrt{2}, \varepsilon_{2} \sqrt{3}, \varepsilon_{3} \sqrt{5}\right): \varepsilon_{i} \in\{ \pm 1\}, \varepsilon_{1} \varepsilon_{2} \varepsilon_{3}=1\right\} .
$$

All pairings between two coördinates are allowed, but there are 4 solutions in total, and not 8 .

Above we have used the linear forms $x_{i}+x_{j}$ to determine the compatibility. Our algorithm uses random linear forms to avoid these problems, or at least make them less probable.

We are now ready to explain the algorithm computing the solutions in number fields that reduce modulo $p^{N}$ to given approximate solutions in $\mathbb{Z}_{p}$. That problem is in fact an instance of the following, more general problem. 
The following algorithm is described as a process that, receiving as input an algebraic system over $\mathbb{Z}$ and an infinite feed of ever-more-precise $p$-adic approximations of a solution, produces a stream of algebraic solutions (in the form of minimal polynomials over $\mathbb{Z}$ and complex numbers singling out roots of the minimal polynomials). The stream eventually exhausts all solutions conjugate to the $p$-adic solution.

Algorithm 6.5 (Solve 0-dimensional algebraic systems). Given: a polynomial system of equations $\mathcal{F}=\left(F_{1}, \ldots, F_{m}\right)$ in variables $x_{1}, \ldots, x_{m}$, having a finite number of solutions; and approximations, to arbitrary precision, of a solution $\left(\widehat{x_{1}}, \ldots, \widehat{x_{m}}\right)$ in $\mathbb{Z}_{p}$

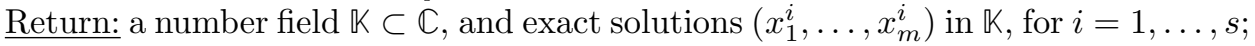
each element of $\mathbb{K}$ is given by its minimal polynomial and an approximation in $\mathbb{C}$ of a particular root.

We construct the solutions $\left(x_{1}^{i}, \ldots, x_{m}^{i}\right)$ iteratively, entry by entry, by constructing partial tables $\left\{\left(x_{1}^{i}, \ldots, x_{k}^{i}\right): i=1, \ldots, s\right\}$. We start by an empty table, with $s=1$ and $k=0$, and let $\epsilon$ be a small number.

Then, for each $k=1, \ldots, m$, we do the following. Using Algorithm 6.1, we compute a likely minimal polynomial $P_{k}$ of $\widehat{x_{k}}$, say of degree $e$. We compute, to precision better than $\epsilon$, approximate roots $r_{1}, \ldots, r_{e} \in \mathbb{C}$ of $P_{k}$. Set

$$
\delta_{1}=\min \left\{\left|r_{i}-r_{j}\right|: 1 \leq i \neq j \leq e\right\} .
$$

If $\delta_{1}<\epsilon$, we halve $\epsilon$ and restart all over.

We next choose randomly a linear form $L=l_{1} x_{1}+\cdots+l_{k} x_{k}$ with $l_{k} \neq 0$. Again using Algorithm 6.1. we compute a minimal polynomial $P_{L}$ for $L\left(\widehat{x_{1}}, \ldots, \widehat{x_{k}}\right)$. If the degree of $P_{L}$ is not divisible by $s$, we choose a different linear form $L$ and repeat the above. Otherwise, we let $\delta_{1}$ be the minimal distance between roots of $P_{L}$. If $\delta_{1}<\epsilon$, we halve $\epsilon$ and restart all over.

We then compute the $s \times e$ matrix $M=\left(M_{i, j}\right)$ with

$$
M_{i, j}= \begin{cases}1 & \text { if }\left|P_{L}\left(L\left(x_{1}^{i}, \ldots, x_{k-1}^{i}, r_{j}\right)\right)\right|<\frac{1}{3} \min \left\{\delta_{1}, \delta_{2}\right\}\|L\|_{1}, \\ 0 & \text { otherwise }\end{cases}
$$

Let the degree of $P_{L}$ be st. If $M$ contains $t$ ones per row and one one per column, then we replace $s$ by $s t$ and replace each row $\left(x_{1}^{i}, \ldots, x_{k-1}^{i}\right)$ is the partial table by $t$ rows $\left(x_{1}^{i}, \ldots, x_{k-1}^{i}, r_{j}\right)$ for all $j$ such that $M_{i, j}=1$. Otherwise, we repeat the step with a different linear form or, if that failed more than ten times in a row, we simply skip the iteration.

When the iteration finished with $k=m$, we have obtained $s$ candidate solutions, which we check algebraically by evaluating $\mathcal{F}$ on them. We output all those that are certifiably valid solutions, and restart the algorithm with better approximations of the $\widehat{x_{1}}, \ldots, \widehat{x_{m}}$.

Proof of validity. First, all the solutions returned are valid, since they were checked (using exact algebra) by evaluating $\mathcal{F}$ on them.

Let now $\left(x_{1}, \ldots, x_{m}\right)$ be a solution that is conjugate to $\left(\widehat{x_{1}}, \ldots, \widehat{x_{m}}\right)$. In particular, the minimal polynomials of the $x_{i}$ and $\widehat{x_{i}}$ are the same, so they will eventually be found by Algorithm 6.1. Similarly, for every linear form $L$ with integer coëfficients, $L\left(x_{1}, \ldots, x_{m}\right)$ and $L\left(\widehat{x_{1}}, \ldots, \widehat{x_{m}}\right)$ also have the same minimal polynomial, so it will also be eventually found by Algorithm 6.1. 
We apply this algorithm to the same polynomial equations (3). The variables $x_{i}$ are a relabeling of the $w_{i, j, s}$ from (4).

\section{Computing The monodromy}

In this section, we detail the second part of the algorithm sketched in $\$ 2$

We are given an approximation of a degree- $d$ rational map $f \in \mathbb{C}(z)$, as well as an approximation of the critical values $Q \subset \mathbb{C}$, and the local degrees $\alpha_{i, 1}, \ldots, \alpha_{i, \ell_{i}}$ above each critical value $Q_{i}$. We are asked to compute the monodromy of the covering induced by $f$.

The first step is to compute a triangulation $\mathscr{Q}$ of $\mathbb{P}^{1}(\mathbb{C})$ by arcs of circle, and containing $Q$ among its vertices. A particularly efficient triangulation is the Delaunay triangulation. This is a decomposition of $\mathbb{P}^{1}(\mathbb{C})$ into triangles, such that, for any two triangles with a common edge, the sum of their opposite angles is $>\pi$. Such a triangulation always exists; is essentially unique; and may be computed e.g. using 20 .

For performance reasons, we refine the triangulation by adding vertices to it: whenever we encounter a triangle whose ratio "circumradius / shortest side" is larger than 1000, we add its circumcenter to the triangulation. This process converges, and gives a reasonably good triangulation in that its triangles are not too acute; see 21.

The dual decomposition $\mathscr{Q}^{\perp}$ of the sphere is the associated Vorono $\ddot{i}$ diagram. It has one vertex, called a dual vertex, per Delaunay triangle and one edge, called dual edge, across every Delaunay edge. Each of its edges $\varepsilon$ is parametrised as the preimage, under a Möbius transformation $\mu_{\varepsilon}$, of the arc $[0,1]$. We denote by $W$ the vertex set of $\mathscr{Q}^{\perp}$, and choose a basepoint $* \in W$. For each $w \in W$, we number arbitrarily the elements of the fibre $f^{-1}(w)$ as $\left\{w_{1}, \ldots, w_{d}\right\}$. Because $W$ is far from $Q$, there are $d$ preimages of each $w \in W$, and their computation is numerically stable.

There are now two strategies, which have both been tested and implemented. The first one is a bit simpler, but the second one performs better in practice. Both associate a permutation $\varsigma_{\varepsilon}$ of $\{1, \ldots, d\}$ with each edge $\varepsilon \in \mathscr{Q}^{\perp}$ from $w^{\prime} \in W$ to $w^{\prime \prime} \in W$, in such a way that the the $f$-lift of $\varepsilon$ that starts at $w_{j}^{\prime}$ ends at $w_{\varsigma_{\varepsilon}(j)}^{\prime \prime}$. Both are explained below; assuming them, we finish the description of the algorithm.

Let $\left(\hat{\gamma}_{i}\right)_{i=1, \ldots, k}$ be non-crossing (but possibly overlapping) paths in $\mathscr{Q}^{\perp}$ that start and end in $*$, cyclically ordered around $*$, such that $\hat{\gamma}_{i}$ surrounds once counterclockwise the point $Q_{i}$ and no other vertex of $Q$. These paths may be selected as follows: choose first the path $\hat{\gamma}_{1}$ arbitrarily, and mark its edges. Then, for $i=2, \ldots, k$, choose the path $\hat{\gamma}_{i}$ in such a manner that it does not cross the previously chosen paths (i.e., it may follow a marked path, but must depart from it on the same side as it joined it), and starts at $*$ in counterclockwise order between the paths $\hat{\gamma}_{i-1}$ and $\hat{\gamma}_{1}$. These paths $\hat{\gamma}_{i}$ are of the following form: follow some edges; then follow counterclockwise the perimeter of the cell of $\mathscr{Q}^{\perp}$ containing $Q_{i}$, i.e. in counterclockwise order the perpendiculars of the edges of $\mathscr{Q}$ touching $Q_{i}$; and then follow in reverse the first edges.

These paths form a basis for the fundamental group $\pi_{1}\left(\mathbb{P}^{1}(\mathbb{C}) \backslash Q, *\right)$, compatible with the description from $\$ 1.1$ Let $\left(\varepsilon_{i, 1}, \ldots, \varepsilon_{i, n_{i}}\right)$ be the edges along $\hat{\gamma}_{i}$, and compute the permutation $\sigma_{i}=\varsigma_{i, 1} \cdots \varsigma_{i, n_{i}}$. Then the monodromy representation of $f$ is given by the family $\left(\sigma_{i}\right)_{i=1, \ldots, k}$. 
7.1. Connect-the-dots. For each dual edge $\varepsilon \in \mathscr{Q}^{\perp}$, going from $w^{\prime}$ to $w^{\prime \prime}$, we do the following. Knowing the spherical distance from $w^{\prime}$ to $w^{\prime \prime}$ and using coarse estimates on $\left|f^{\prime}(z)\right|$, we have an upper bound on the length of each of the $d$ preimages of $\varepsilon$. We attempt to match each $w_{j}^{\prime}$ with a $w_{\varsigma(j)}^{\prime \prime}$ for some permutation $\varsigma \in \mathfrak{S}_{d}$, by matching each $w_{j}^{\prime}$ to the closest $w_{\varsigma(j)}^{\prime \prime}$. If more than one match is compatible with the upper bound on the length of an arc above the arc from $w^{\prime}$ to $w^{\prime \prime}$, we subdivide the edge $\varepsilon$.

Algorithm 7.1 (Lifting edges by connect-the-dots).

Given: a rational map $f \in \mathbb{C}(z)$, an edge $\varepsilon=\mu_{\varepsilon}^{-1}[0,1]$ from $w^{\prime}=\mu_{\varepsilon}^{-1}(0)$ to $\overline{w^{\prime \prime}}=\varepsilon^{-1}(1)$ and orderings $w_{1}^{\prime}, \ldots, w_{d}^{\prime}$ and $w_{1}^{\prime \prime}, \ldots, w_{d}^{\prime \prime}$ of the preimages of $w^{\prime}, w^{\prime \prime}$ respectively

Return: a permutation $\varsigma \in \mathfrak{S}_{d}$ such that the $f$-lift of $\varepsilon$ starting at $w_{j}^{\prime}$ ends at $w_{\varsigma(j)}^{\prime \prime}$

More generally, the algorithm computes, for arbitrary $0 \leq s<t \leq 1$, the matching between $f$-preimages of $\mu_{\varepsilon}^{-1}(s)$ and of $\mu_{\varepsilon}^{-1}(t)$; the solution is provided by the matching for $s=0$ and $t=1$. We write $\left\{w_{j}(t)\right\}_{j=1, \ldots, d}$ for the $f$-preimages of $\mu_{\varepsilon}^{-1}(t)$.

If there is only one possible match between the sets $\left\{w_{j}(s)\right\}$ and $\left\{w_{j}(t)\right\}$, given by a permutation $\varsigma$, then the algorithm returns that permutation. Otherwise, set $u=(s+t) / 2$, compute the preimages $\left\{w_{j}(u)\right\}_{j=1, \ldots, d}$ of $\mu_{\varepsilon}^{-1}(u)$, recursively compute the matching between the $w_{j}(s)$ and $w_{j}(u)$ and between the $w_{j}(u)$ and $w_{j}(t)$, and return the product of the corresponding permutations.

7.2. Using two triangulations. The second algorithm is more efficient, and uses fundamentally the fact that the arcs in the triangulation $\mathscr{Q}^{\perp}$ and in its $f$-preimage are given by algebraic curves.

We initially compute the Delaunay triangulation $\mathscr{C}$ on $f^{-1}(Q)$, and parametrise its edges $e$ via Möbius transformations $\nu_{e}$ such that $e=\nu_{e}^{-1}([0,1])$.

It is straightforward to lift $\mathscr{Q}^{\perp}$ through $f$ : its edges are all the curves defined by equations $\left(\mu_{\varepsilon} \circ f\right)(z) \in[0,1]$.

Algorithm 7.2 (Lifting edges using two triangulations).

Given: a rational map $f \in \mathbb{C}(z)$, an edge $\varepsilon=\mu_{\varepsilon}^{-1}[0,1]$ from $w^{\prime}=\mu_{\varepsilon}^{-1}(0)$ to $\overline{w^{\prime \prime}}=\varepsilon^{-1}(1)$ and orderings $w_{1}^{\prime}, \ldots, w_{d}^{\prime}$ and $w_{1}^{\prime \prime}, \ldots, w_{d}^{\prime \prime}$ of the preimages of $w^{\prime}, w^{\prime \prime}$ respectively

Return: a permutation $\varsigma \in \mathfrak{S}_{d}$ such that the $f$-lift of $\varepsilon$ starting at $w_{j}^{\prime}$ ends at $w_{\varsigma(j)}^{\prime \prime}$

For each $i=1, \ldots, d$, we seek the $j \in\{1, \ldots, d\}$ such that the lift of $\varepsilon$ starting at $w_{i}^{\prime}$ ends at $w_{j}^{\prime \prime}$. The permutation to return is then the map $i \mapsto j$.

We first determine in which triangle $T$ of $\mathscr{C}$ the lift $w_{i}^{\prime}$ lies. Then we compute whether the lift $\tilde{\varepsilon}$ of $\varepsilon$ starting at $w_{i}^{\prime}$ leaves $T$. If this happens, then it must cross an edge $e$ of $T$, namely, we have $\mu_{\varepsilon} \circ f \circ \nu_{e}^{-1}([0,1]) \in[0,1]$. This entails, firstly, that the imaginary part of $\mu_{\varepsilon} \circ f \circ \mu_{e}^{-1}$ vanishes, and secondly that its real part belongs to $[0,1]$. Both are polynomial conditions imposed on real-valued polynomials, and are efficiently computable numerically. We also keep track of the point of intersection $\tilde{w}_{e}$ of $\tilde{\varepsilon}$ and $e$.

In that case, we move to the neighbouring triangle $T^{\prime}$ of $T$ along edge $e$, and continue. When we do not detect more intersections with edges of $\mathscr{C}$, we know in which triangle of $\mathscr{C}$ the vertex $w_{j}^{\prime \prime}$ lies.

It may happen that two or more vertices $w_{j}^{\prime \prime}$ belong to the same triangle $T$ that we have found in the previous paragraph. In that case, we let $\tilde{w}$ denote the last 
point on $\tilde{\varepsilon}$ that was computed - possibly $w_{i}^{\prime}$; it also belongs to $T$. We consider in turn all candidates $w_{j}^{\prime \prime}$, and compute the straight path $\delta_{j}$ from $\tilde{w}$ to $w_{j}^{\prime \prime}$ and its image $f\left(\delta_{j}\right)$. If there exists a unique $j$ such that $f\left(\delta_{j}\right)$ lies in the two triangles of $\mathscr{Q}$ to which $\varepsilon$ belongs, then we have found the desired $j$. If there are no such $j$, then we interpolate. Let $t_{0} \in[0,1]$ be such that $f(\tilde{w})=\varepsilon\left(t_{0}\right)$. Consider $t \in\left(t_{0}, 1\right)$, and those lifts $w \in f^{-1}(\varepsilon(t))$ that belong to $T$; we then consider the paths $\delta_{j}$ from $w$ to $w_{j}^{\prime \prime}$ as before, and continue with increasing $t$.

Additional care must be taken for tangent crossings of edges (when the imaginary part of $\mu_{\varepsilon} \circ f \circ \mu_{e}^{-1}$ has a multiple zero), and when vertices of $\mathscr{C}$ lie on edges of $\mathscr{Q}^{\perp}$ or conversely; these are treated as special cases. However, because of the necessary crudeness of norm estimates on $\left|f^{\prime}(z)\right|$ in the first method, this second method is preferable.

\section{An APPLICATION TO DYNAMICAL SYSTEMS}

We recall from the introduction that the post-critical set of a branched selfcovering $f: \mathbb{S} \rightarrow \mathbb{S}$ with critical value set $Q_{f}$ is

$$
P_{f}:=\bigcup_{n \geq 0} f^{\circ n}\left(Q_{f}\right) \text {. }
$$

We are interested in the case where $P_{f}$ is finite and we consider $f$ up to isotopy rel $P_{f}$; namely, $f \sim g$ if there exists a path of branched self-coverings from $f$ to $g$ whose post-critical set moves smoothly. We say that $f$ is combinatorially equivalen ${ }^{1}$ to a rational map $F$ if there are orientation-preserving homeomorphisms $\phi: \mathbb{S} \rightarrow \mathbb{P}^{1}(\mathbb{C})$ and $\psi: \mathbb{S} \rightarrow \mathbb{P}^{1}(\mathbb{C})$ such that $F \circ \phi=\psi \circ f$ and $\phi$ is isotopic to $\psi$ rel $P_{f}$.

On the one hand, many examples of branched self-coverings can be constructed combinatorially, via triangulations; for these, it is natural to consider the maps up to isotopy rel the vertices of the triangulation. On the other hand, a fundamental theorem by Thurston points to the rigidity of these objects:

Theorem 8.1 (Thurston; see 7]). Let $f: \mathbb{S} \rightarrow \mathbb{S}$ be branched self-covering with finite post-critical set $P_{f}$. For each $p \in P_{f}$, set $o_{p}=\operatorname{ppcm}\left\{\operatorname{deg}_{q}\left(f^{n}\right): q \in\right.$ $\left.f^{-n}(p), n>0\right\} \in \mathbb{N} \cup\{\infty\}$, and assume that $\sum_{p \in P_{f}}\left(1-1 / o_{p}\right)>2$. This condition is usually abbreviated into " $f$ has hyperbolic orbispace".

Then $f$ is combinatorially equivalent to a rational map if and only if $f$ admits no "Thurston obstruction", namely, if and only if, for every collection $\mathcal{C}$ of isotopy classes of non-peripheral disjoint curves on $\mathbb{S} \backslash P_{f}$, the $\mathbb{Q C}$-endomorphism

$$
\mathcal{C} \ni c \mapsto \sum_{d \in f^{-1}(c) \cap \mathcal{C}} \frac{1}{\operatorname{deg}(f: d \rightarrow c)} \cdot d \in \mathbb{Q} \mathcal{C}
$$

has spectral radius $<1$.

Furthermore, in that case, the rational map is unique up to conjugation by a Möbius transformation.

\footnotetext{
${ }^{1}$ This is sometimes called Thurston equivalence
} 
8.1. Cui's Problem. Cui Guizhen suggested in 2010 that if $f$ is a "Sierpiński map", namely a rational map whose Julia set is a Sierpiński carpet, then there should exist an essential, non-peripheral, simple curve $\gamma$ such that $f^{-n}(\gamma)$ contains at least two components homotopic to $\gamma$ rel $P_{f}$, for some $n$ large enough. He then found a counterexample to his suggestion, given combinatorially as follows:

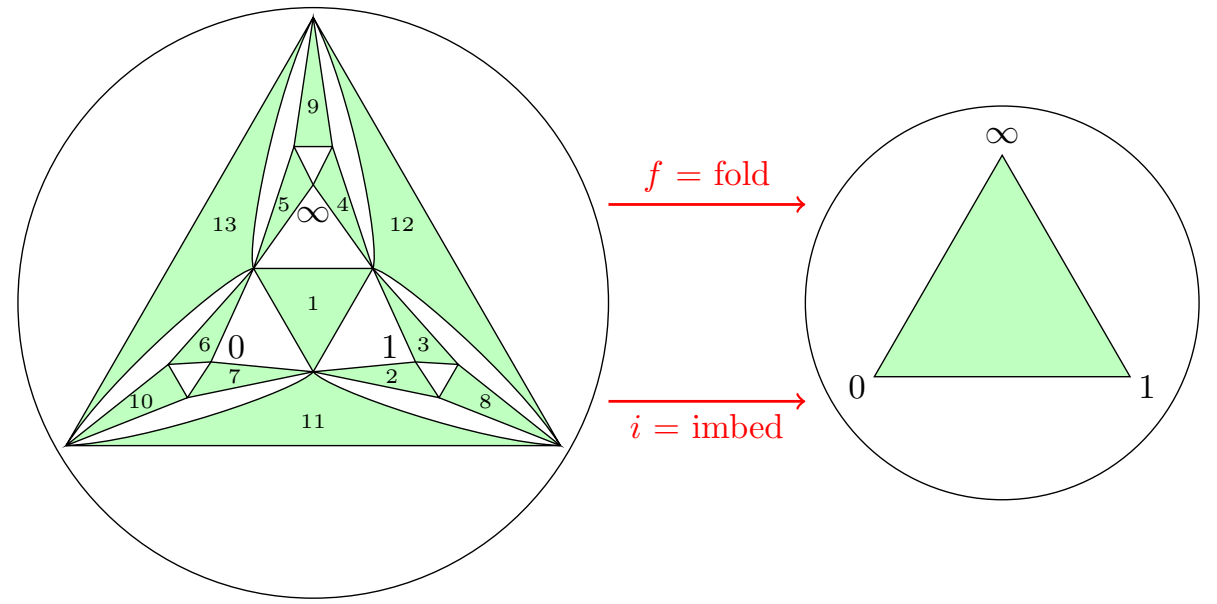

In that case, $P_{f}=Q:=\left\{Q_{1}, Q_{2}, Q_{3}\right\}$ with $Q_{1}:=\infty, Q_{2}:=0$ and $Q_{3}:=1$, and $f$ fixes $P_{f}$ pointwise. Since $f$ has only three post-critical points, all curves are peripheral. On the other hand, the Julia set of $f$ is a Sierpiński carpet, as we now show. For all $i \in\{1,2,3\}$, let $\mathcal{U}_{i}$ be the immediate basin of $Q_{i}$. Given $i, j \in\{1,2,3\}$ not necessarily distinct, there exists up to isotopy a unique properly embedded arc with endpoints at $Q_{i}, Q_{j}$ whose interior avoids $P_{f}$. Direct inspection of the triangulation shows that none of these arcs are invariant under $f$ up to isotopy. From this it follows that the closures of the $\mathcal{U}_{i}$ are pairwise disjoint, and that the boundary of each $\mathcal{U}_{i}$ is a Jordan domain. Consider next the preimages of the basins $\mathcal{U}_{i}$. Using the fact that $f$ is hyperbolic, no branching occurs on their boundaries, so all iterated $f$-preimages of the $\mathcal{U}_{i}$ have disjoint closures and the Julia set is a Sierpiński carpet as claimed.

Kevin Pilgrim indicated to us a degree-3 rational map exhibiting the same phenomenon (its Julia set is a Sierpiński carpet, and it contains no self-replicating multicurve): start by the degree-2 rational map coming from the Torus endomorphism $z \mapsto(1+i) z$ on $\mathbb{C} / \mathbb{Z}+i \mathbb{Z}$ via the Weierstrass map $\wp$. Then blow up the edge between $\wp(0)$ and the fixed point.

From the above picture, it is easy to compute the monodromy action about $Q$ : the permutations are those given in (2), namely

$$
\begin{aligned}
& \sigma_{1}=(1,7,11,2)(3,8) \underline{(4,5)}(6,10)(9,12,13), \\
& \sigma_{2}=(1,3,12,4)(5,9) \underline{(6,7)}(10,13,11)(2,8), \\
& \sigma_{3}=(1,5,13,6)(7,10) \underline{(2,3)}(8,11,12)(4,9) .
\end{aligned}
$$

Furthermore, the underlined cycles mark which preimage of a critical value should be fixed. This extra dynamical data is required to determine the combinatorial equivalence class of $f$, and it is also sufficient since $\# P_{f}=3$ so the pure mapping class group of $\left(\mathbb{P}^{1}(\mathbb{C}), \# P_{f}\right)$ is trivial. The orbispace of $f$ is hyperbolic, because 
$o_{p}=\infty$ for each $p \in P_{f}$. Because $\# P_{f}=3$, all curves on $\mathbb{P}^{1}(\mathbb{C}) \backslash P_{f}$ are peripheral, so no Thurston obstruction may occur. By Theorem 8.1, there is then, up to Möbius conjugacy, a unique map with monodromy $\left(\sigma_{1}, \sigma_{2}, \sigma_{3}\right)$, fixing $\infty, 0,1$ with local degree 2 , and such that $\infty, 0,1$ are critical points marked by the cycles $(4,5),(6,7),(2,3)$ respectively.

Therefore, the map computed by our algorithm, after precomposition with a suitable Möbius transformation that puts the preimages of $Q$ at the points determined by the cycles $(4,5),(6,7),(2,3)$ respectively, is the required solution.

Our algorithm searched in fact for a map $F$ with $\infty, 0,1$ of order 4 . This is an improvement to searching immediately for the correct map, because there are three points of order 2 above each of $\infty, 0,1$, and they may lie in a strict field extension.

It remains to determine the appropriate Möbius transformation with which to precompose $F$. The first author developed an algorithm that determines, from a rational map given by its coëfficients, both the monodromy about the critical values and the identification of critical points with cycles of the monodromy permutations. This algorithm is part of the software package IMG within the computer algebra system GAP [8], and will be described elsewhere. Note, however, that there are finitely many possibilities to consider for the sought Möbius transformation, and the correct one can be found by inspection. To find the appropriate one and thus determine the solution to Cui's problem, it suffices to draw the preimage of the upper hemisphere under $F$, and to identify on the picture the appropriate preimages of $\infty, 0,1$. In the image below, $F$ was normalized so that the order- 4 critical points above $\infty, 0,1$ are at cube roots of unity $1, \omega, \omega^{2}$ respectively. The appropriate preimages of $\infty, 0,1$ are marked by a small red circle, based on the figure above:

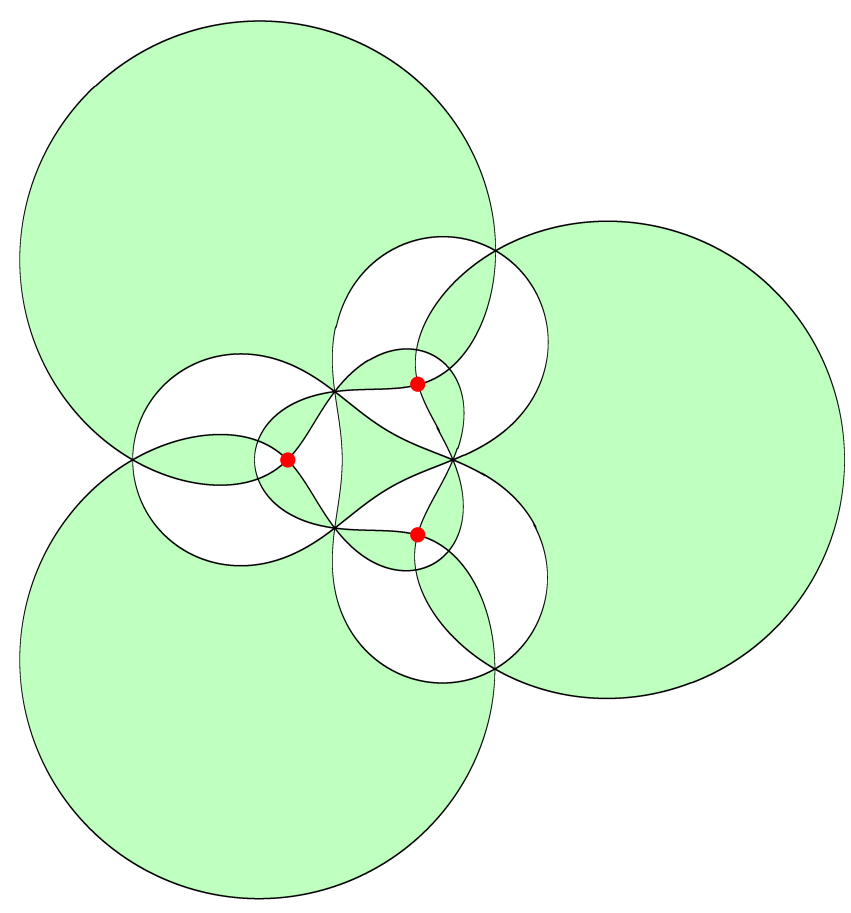


Our algorithm found a solution $(\bmod 11)$ of the defining equations for a map; then lifted them $\left(\bmod 11^{2^{6}}\right)$ and finally obtained six Galois conjugate solutions. The correct one (with correct choice of point of order 2 above $\infty, 0,1$ ) was then found. The original map is of the form

$$
f(z / w)=\lambda \frac{\left(z-b_{4} w\right)^{4}\left(z-b_{3} w\right)^{3}\left(z-b_{2,1} w\right)^{2}\left(z-b_{2,2} w\right)^{2}\left(z-b_{2,3} w\right)^{2}}{\left(z-a_{4} w\right)^{4}\left(z-a_{3} w\right)^{3}\left(z-a_{2,1} w\right)^{2}\left(z-a_{2,2} w\right)^{2}\left(z-a_{2,3} w\right)^{2}}
$$

here are the preimages $a_{i}, b_{i}, c_{i}$ of $\infty, 0,1$ respectively:

$$
\begin{aligned}
a_{4} & \left.=\infty \text { (meaning the term } z-a_{4} w \text { should be replaced by } 1\right) \\
a_{3} & \approx 0.500000000000000000000000000000-0.439846359796987134487167714627 i, \\
a_{2,1} & \approx 1.61268567872451072013417667720-0.490182463946729812334860743821 i, \\
a_{2,2} & \approx 0.500000000000000000000000000000-0.0415300696430258467988035191529 i, \\
a_{2,3} & \approx-0.612685678724510720134176677204-0.490182463946729812334860743821 i, \\
b_{4} & =0 \\
b_{3} & \approx 1.12748515145901194873474709466-0.991840479188802206853242764751 i, \\
b_{2,1} & \approx 1.98629656633071582984701575517-0.164982069462835473582606346591 i, \\
b_{2,2} & \approx 0.567640411622375679553529964298-0.172536644477962176299255320022 i, \\
b_{2,3} & \approx-0.995164705141609432502666361446-0.796186860797306011242450678339 i, \\
c_{4} & =1, \\
c_{3} & \approx-0.127485151459011948734747094655-0.991840479188802206853242764751 i, \\
c_{2,1} & \approx 0.432359588377624320446470035702-0.172536644477962176299255320022 i, \\
c_{2,2} & \approx-0.986296566330715829847015755165-0.164982069462835473582606346591 i, \\
c_{2,3} & \approx 1.99516470514160943250266636145-0.796186860797306011242450678339 i, \\
\lambda & \approx 0.130027094895701439414281708196 i
\end{aligned}
$$

The required Möbius transformation $\mu$ maps $(\infty, 0,1)$ to $\left(a_{2,2}, b_{2,3}, c_{2,2}\right)$ respectively, so

$$
\mu(z)=\frac{a_{2,2}\left(c_{2,2}-b_{2,3}\right) z+b_{2,3}\left(a_{2,2}-c_{2,2}\right)}{\left(c_{2,2}-b_{2,3}\right) z+\left(a_{2,2}-c_{2,2}\right)}
$$

and the required solution is $f \circ \mu$. Its Julia set is displayed in Figure 1

Acknowledgments. We are grateful to Kevin Pilgrim for valuable remarks on a preliminary version of the text, and for more examples of Sierpinski maps with three post-critical points. The referee pointed out with great acuity some deficiencies in the exposition, and contributed a shorter proof of the assertion that the Julia set of Cui's map is a Sierpiński carpet.

\section{REFERENCES}

[1] A. Oliver L. Atkin and H. Peter F. Swinnerton-Dyer, Modular forms on noncongruence subgroups, Combinatorics (Proc. Sympos. Pure Math., Vol. XIX, Univ. California, Los Angeles, Calif., 1968), Amer. Math. Soc., Providence, R.I., 1971, pp. 1-25. MR0337781 (49 \#2550)

[2] PARI/GP, version 2.5.0, The PARI Group, Bordeaux, 2011.

[3] Philip L. Bowers and Kenneth Stephenson, Uniformizing dessins and Belyॅ maps via circle packing, Mem. Amer. Math. Soc. 170 (2004), no. 805, xii+97. MR2053391 (2005a:30068)

[4] Suzanne Hruska Boyd and Christian Henriksen, The Medusa algorithm for polynomial matings, Conform. Geom. Dyn. 16 (2012), 161-183, DOI 10.1090/S1088-4173-2012-00245-7. MR2943594 


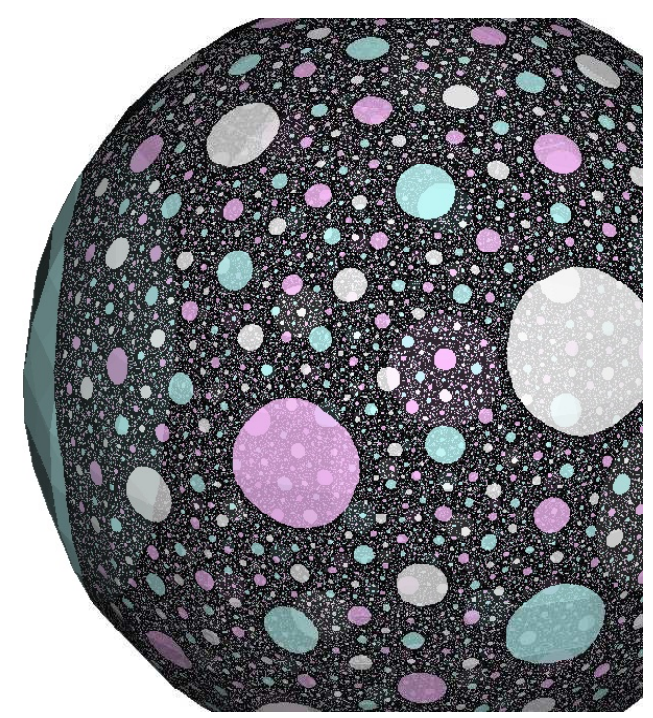

Figure 1. Julia set of Cui's map

[5] Jean-Marc Couveignes and Louis Granboulan, Dessins from a geometric point of view, The Grothendieck theory of dessins d'enfants (Luminy, 1993), London Math. Soc. Lecture Note Ser., vol. 200, Cambridge Univ. Press, Cambridge, 1994, pp. 79-113. MR1305394 (96b:14015)

[6] Jean-Marc Couveignes, Quelques revêtements définis sur $\mathbb{Q}$, Manuscripta Math. 92 (1997), no. 4, 409-445, DOI 10.1007/BF02678203 (French, with French summary). MR1441485 (98c:14021)

[7] Adrien Douady and John H. Hubbard, A proof of Thurston's topological characterization of rational functions, Acta Math. 171 (1993), no. 2, 263-297. MR1251582 (94j:58143)

[8] The GAP Group, GAP - Groups, Algorithms, and Programming, Version 4.4.10, 2008.

[9] Alexandre Grothendieck, Esquisse d'un programme, Geometric Galois actions, 1, London Math. Soc. Lecture Note Ser., vol. 242, Cambridge Univ. Press, Cambridge, 1997, pp. 5-48 (French, with French summary). With an English translation on pp. 243-283. MR1483107 (99c:14034)

[10] John H. Hubbard and Dierk Schleicher, The spider algorithm, Complex dynamical systems (Cincinnati, OH, 1994), Proc. Sympos. Appl. Math., vol. 49, Amer. Math. Soc., Providence, RI, 1994, pp. 155-180. MR1315537

[11] Adolf Hurwitz, Ueber Riemann'sche Flächen mit gegebenen Verzweigungspunkten, Math. Ann. 39 (1891), no. 1, 1-60, DOI 10.1007/BF01199469 (German). MR1510692

[12] R. Kühnau, Numerische Realisierung konformer Abbildungen durch "Interpolation", Z. Angew. Math. Mech. 63 (1983), no. 12, 631-637, DOI 10.1002/zamm.19830631206 (German, with English and Russian summaries). MR737000 (85b:30012)

[13] Arjen K. Lenstra, Hendrik W. Lenstra Jr., and László Lovász, Factoring polynomials with rational coefficients, Math. Ann. 261 (1982), no. 4, 515-534, DOI 10.1007/BF01457454. MR682664 (84a:12002)

[14] Gunter Malle, Polynomials for primitive nonsolvable permutation groups of degree $d \leq 15$, J. Symbolic Comput. 4 (1987), no. 1, 83-92, DOI 10.1016/S0747-7171(87)80056-1. MR908415 (89b:12007)

[15] Gunter Malle and Bernd H. Matzat, Realisierung von Gruppen $\operatorname{PSL}_{2}\left(\mathbf{F}_{p}\right)$ als Galoisgruppen über Q, Math. Ann. 272 (1985), no. 4, 549-565, DOI 10.1007/BF01455866 (German). MR807290 (87e:12002)

[16] Donald E. Marshall and Steffen Rohde, Convergence of a variant of the zipper algorithm for conformal mapping, SIAM J. Numer. Anal. 45 (2007), no. 6, 2577-2609 (electronic), DOI 10.1137/060659119. MR2361903 (2008m:30008) 
[17] Yu. V. Matiyasevich, Computation of generalized Chebyshev polynomials on a computer, Vestnik Moskov. Univ. Ser. I Mat. Mekh. 6 (1996), 59-61, 112 (Russian, with Russian summary); English transl., Moscow Univ. Math. Bull. 51 (1996), no. 6, 39-40 (1997). MR1481512

[18] Kevin M. Pilgrim, Dessins d'enfants and Hubbard trees, Ann. Sci. École Norm. Sup. (4) 33 (2000), no. 5, 671-693, DOI 10.1016/S0012-9593(00)01050-8 (English, with English and French summaries). MR1834499 (2002m:37062)

[19] Alfredo Poirier, Critical portraits for postcritically finite polynomials, Fund. Math. 203 (2009), no. 2, 107-163, DOI 10.4064/fm203-2-2. MR2496235 (2010c:37095)

[20] Robert J. Renka, Algorithm 772: STRIPACK: Delaunay triangulation and Voronoi diagram on the surface of a sphere, ACM Trans. Math. Software 23 (1997), no. 3, 416-434, DOI 10.1145/275323.275329. MR1672176

[21] Jonathan R. Shewchuk, Delaunay refinement algorithms for triangular mesh generation, Comput. Geom. 22 (2002), no. 1-3, 21-74, DOI 10.1016/S0925-7721(01)00047-5. 16th ACM Symposium on Computational Geometry (Hong Kong, 2000). MR1893652 (2003b:65131)

L.B.: Mathematisches Institut, Georg-August Universität zu Göttingen

X.B.: Institut de Mathématiques de Toulouse, Université Paul Sabatier, Toulouse

H-.C.G.v.B., J.K.: Courant Research Centre "Higher Order Structures", GeorgAugust Universität ZU Göttingen 